JOURNAL OF THE

AMERICAN MATHEMATICAL SOCIETY

Volume 24, Number 4, October 2011, Pages 919-944

S 0894-0347(2011)00703-0

Article electronically published on May 6, 2011

\title{
BETA ENSEMBLES, STOCHASTIC AIRY SPECTRUM, AND A DIFFUSION
}

\author{
JOSÉ A. RAMÍREZ, BRIAN RIDER, AND BÁLINT VIRÁG
}

\section{INTRODUCTION}

For any $\beta>0$, consider the probability density function of $\lambda_{1} \geq \lambda_{2} \geq \cdots \geq \lambda_{n}$ $\in \mathbb{R}$ given by

$$
\mathbb{P}_{n}^{\beta}\left(\lambda_{1}, \lambda_{2}, \ldots, \lambda_{n}\right)=\frac{1}{Z_{n}^{\beta}} e^{-\beta \sum_{k=1}^{n} \lambda_{k}^{2} / 4} \prod_{j<k}\left|\lambda_{j}-\lambda_{k}\right|^{\beta},
$$

in which $Z_{n}^{\beta}$ is a normalizing constant. When $\beta=1,2$ or 4 this is the joint density of eigenvalues for the Gaussian orthogonal, unitary, or symplectic ensembles, $\mathrm{G}(\mathrm{O} / \mathrm{U} / \mathrm{S}) \mathrm{E}$, of random matrix theory. For these special values of $\beta$, the above model is solvable: all finite-dimensional correlation functions may be computed explicitly in terms of Hermite functions, allowing for a startling collection of precise local limit theorems for the random points (see [7], 8] for background).

The law (1.1) also describes a one-dimensional Coulomb gas at inverse temperature $\beta$ and thus is of physical interest. In fact, (1.1) is intimately connected to Calogero-Sutherland quantum systems and hence to Jack polynomials (an important system of multiple orthogonal polynomials). Still, despite long being the focus of several branches of research, there are no known forms of the $\beta \neq 1,2,4$ correlations which appear amenable to asymptotics. (The comprehensive text [20] contains an account of the previous general-beta developments.)

It was therefore welcome news when, based on 31, Dumitriu and Edelman [12 discovered the following family of matrix models for all $\beta$. Let $g_{1}, g_{2}, \ldots, g_{n}$ be independent Gaussians with mean 0 and variance 2 . Also let $\chi_{\beta}, \chi_{2 \beta}, \ldots, \chi_{(n-1) \beta}$ be independent $\chi$ random variables indexed by the shape parameter. Then the $n$ eigenvalues of the tridiagonal matrix ensemble

$$
H_{n}^{\beta}=\frac{1}{\sqrt{\beta}}\left[\begin{array}{ccccc}
g_{1} & \chi_{(n-1) \beta} & & & \\
\chi_{(n-1) \beta} & g_{2} & \chi_{(n-2) \beta} & & \\
& \ddots & \ddots & \ddots & \\
& & \chi_{2 \beta} & g_{n-1} & \chi_{\beta} \\
& & & \chi_{\beta} & g_{n}
\end{array}\right]
$$

Received by the editors November 3, 2009 and, in revised form, November 9, 2010. 2010 Mathematics Subject Classification. Primary 60F05, 60H25.

Key words and phrases. Random matrices, Tracy-Widom laws, random Schrödinger.

The second author was supported in part by NSF grants DMS-0505680 and DMS-0645756

The third author was supported in part by a Sloan Foundation fellowship, by the Canada Research Chair program, and by NSERC and Connaught research grants.

(C)2011 American Mathematical Society Reverts to public domain 28 years from publication 
have joint law given by (1.1). These are more specifically referred to as the $\beta$ Hermite ensembles.

We focus on the implications of this discovery to the point process limits of the spectral edge in the general $\beta$-ensembles. The distributional limits of the largest eigenvalues in $\mathrm{G}(\mathrm{O} / \mathrm{U} / \mathrm{S}) \mathrm{E}$ comprise some of the most celebrated results in random matrix theory due to their surprising importance in physics, combinatorics, multivariate statistics, engineering, and applied probability: [1, 4], [19], 22], 23], and [26] mark a few highlights. The basic result is: for $\beta=1,2$, or 4 and $n \uparrow \infty$, centered by $2 \sqrt{n}$ and scaled by $n^{1 / 6}$, the largest eigenvalue converges in law to the $\operatorname{Tracy}-\operatorname{Widom}(\beta)$ distribution (see [29] and 30]), which is given explicitly in terms of the second Painlevé transcendent. There are allied results for second, third, etc. eigenvalues; see again [29] as well as [10].

The wide array of models for which Tracy-Widom describes the limit statistics identifies these laws as important new probability distributions. Still, our understanding of these laws is in its infancy. It remains desirable to obtain a set of characterizing conditions, such as those classically known for say the Gaussian or Poisson laws. A description of the limit distribution of the largest eigenvalues in the general $\beta$-Hermite ensembles is a first step, providing additional information on the structure of the three Tracy-Widom laws via the structure of a one-parameter family of distributions in which they naturally reside.

Towards an edge limit theorem at general $\beta$, Sutton 28 and Edelman and Sutton [15] present a promising heuristic argument that the rescaled operators

$$
\tilde{H}_{n}^{\beta}=n^{1 / 6}\left(2 \sqrt{n} I-H_{n}^{\beta}\right),
$$

where $I$ is the $n \times n$ identity, should correspond to

$$
\mathcal{H}_{\beta}=-\frac{d^{2}}{d x^{2}}+x+\frac{2}{\sqrt{\beta}} b_{x}^{\prime}
$$

in the $n \uparrow \infty$, or continuum, limit. Here $b^{\prime}$ indicates a white noise, and the proposed scaling of the matrix ensembles follows the edge scaling in the known cases. Thus, were it to hold, the above correspondence would entail that the low-lying eigenvalues of $\tilde{H}_{n}^{\beta}$ converge in law to those of $\mathcal{H}_{\beta}$. Our first result is a proof of this heuristic.

In Section 2 we give a precise definition of this limiting "stochastic Airy operator" $\left(\mathrm{SAE}_{\beta}\right)$. For now, let $L^{*}$ denote the space of functions $f$ satisfying $f(0)=0$ and $\int_{0}^{\infty}\left(f^{\prime}\right)^{2}+(1+x) f^{2} d x<\infty$. Then we say that $(\psi, \lambda) \in L^{*} \times \mathbb{R}$ is an eigenfunction/eigenvalue pair for $\mathcal{H}_{\beta}$ if $\|\psi\|_{2}=1$ and

$$
\psi^{\prime \prime}(x)=\frac{2}{\sqrt{\beta}} \psi(x) b_{x}^{\prime}+(x-\lambda) \psi(x)
$$

holds in the following integration-by-parts sense:

$$
\psi^{\prime}(x)-\psi^{\prime}(0)=\frac{2}{\sqrt{\beta}} \psi(x) b_{x}+\int_{0}^{x}-\frac{2}{\sqrt{\beta}} b_{y} \psi^{\prime}(y) d y+\int_{0}^{x}(y-\lambda) \psi(y) d y,
$$

where all integrands are products of locally $L^{2}$ functions. The set of eigenvalues is then a deterministic function of the random Brownian path $b$.

Theorem 1.1. With probability one, for each $k \geq 0$ the set of eigenvalues of $\mathcal{H}_{\beta}$ has a well-defined $(k+1)$ st lowest element $\Lambda_{k}$. Moreover, let $\lambda_{1} \geq \lambda_{2} \geq \cdots$ denote 
the eigenvalues of the Hermite $\beta$-ensemble $H_{n}^{\beta}$. Then the vector

$$
\left(n^{1 / 6}\left(2 \sqrt{n}-\lambda_{\beta, \ell}\right)\right)_{\ell=1, \ldots, k}
$$

converges in distribution as $n \rightarrow \infty$ to $\left(\Lambda_{0}, \Lambda_{1}, \ldots, \Lambda_{k-1}\right)$.

We mention that though the $n^{1 / 6}$ scaling across all beta was anticipated from the Tracy-Widom results at $\beta=1,2,4$, previous authors had only obtained bounds on this rate. For instance, [1] proves that the fluctuations of $\lambda_{\beta, 1}$ are no greater than $n^{1 / 2}$ while, for even integer values of beta, 9] contains a suggestive calculation on the one-point function in a vicinity of the edge which produces the correct order.

The proof of Theorem 1.1 relies on an equivalent variational formulation of the eigenvalue problem. As we shall show: with self-evident notation,

$$
T W_{\beta}=\sup _{f \in L^{*},\|f\|_{2}=1}\left\{\frac{2}{\sqrt{\beta}} \int_{0}^{\infty} f^{2}(x) d b(x)-\int_{0}^{\infty}\left[f^{\prime}(x)^{2}+x f^{2}(x)\right] d x\right\},
$$

where the stochastic integral is again defined via an integration by parts procedure. The equality here is in law, and one may view this as a definition of $T W_{\beta}$, independent of any random matrix theory developments. This variational approach actually allows for a type of universality result, and we provide rather general conditions under which the spectrum of random tridiagonal models converges to that of their natural continuum operator limit. In particular, one can obtain the TracyWidom laws by considering tridiagonal matrix versions of (1.4) far simpler than (1.3).

Our next theorem gives yet another characterization of the limiting spectrum in terms of the explosion probability of the one-dimensional diffusion $x \mapsto p(x)$ defined by the Itô equation

$$
d p(x)=\frac{2}{\sqrt{\beta}} d b_{x}+\left(x-p^{2}(x)\right) d x .
$$

Theorem 1.2. Let $\kappa(x, \cdot)$ be the distribution of the first time passage to $-\infty$ of the diffusion $p(x)$ when started from $+\infty$ at time $x$. Then

$$
\begin{aligned}
& \mathbb{P}\left(\Lambda_{0}>\lambda\right)=\kappa(-\lambda,\{\infty\}), \quad \text { and, for } k \geq 0, \\
& \mathbb{P}\left(\Lambda_{k}<\lambda\right)=\int_{\mathbb{R}^{k+1}} \kappa\left(-\lambda, d x_{1}\right) \kappa\left(x_{1}, d x_{2}\right) \ldots \kappa\left(x_{k}, d x_{k+1}\right) .
\end{aligned}
$$

Even in the well-understood $\beta=1,2$, and 4 settings, any such simple Markovian description of $T W_{\beta}$ is novel. Further, a variant of (1.8) is shown to describe joint laws in Proposition 3.4 .

Theorem 1.2 combined with the variational picture leads to our final result on the shape of the general beta laws, so far only known for $\beta=1,2,4$. (In fact, for $\beta=1,2,4$ these type of asymptotics are known down to the constant order terms; see for example [3. In addition, after the completion of this paper there has been further progress on the right tail estimate for general $\beta$; see [14].)

Theorem 1.3. With $T W_{\beta}=-\Lambda_{0}(\beta)$, for $a \uparrow \infty$ we have

$$
\begin{aligned}
\mathbb{P}\left(T W_{\beta}>a\right) & =\exp \left(-\frac{2}{3} \beta a^{3 / 2}(1+o(1))\right), \quad \text { and } \\
\mathbb{P}\left(T W_{\beta}<-a\right) & =\exp \left(-\frac{1}{24} \beta a^{3}(1+o(1))\right) .
\end{aligned}
$$


As indicated, the above discussion carries over to a large class of $\beta$-ensembles. For example, let $W$ be an $n \times \kappa$ matrix comprised of independent standard real, complex, or quaternion Gaussians. Viewing $W$ as $\kappa$ random vectors, the sample covariance matrix $W W^{\dagger}$ (known as the Laguerre, or Wishart ensemble for $\beta=1,2,4$ ) plays an important role in mathematical statistics.

Johansson, in the complex case 22], and Johnstone, in the real case 23, showed that the largest eigenvalues tend to the $\beta=2$ and $\beta=1$ Tracy-Widom laws whenever $\kappa / n \rightarrow \vartheta \in(0, \infty)$. Later, El Karoui [16] showed that the same result holds even if $\kappa / n \rightarrow \infty$ or 0 if both $n, \kappa \rightarrow \infty$, this regime being important in applications. Our proof handles all regimes simultaneously for all $\beta$.

To explain, now consider the joint density on points $\lambda_{1}, \ldots, \lambda_{n} \in \mathbb{R}^{+}$,

$$
\mathbb{P}_{n, \kappa}^{\beta}\left(\lambda_{1}, \lambda_{2}, \ldots, \lambda_{n}\right)=\frac{1}{Z_{n, \kappa}^{\beta}} \prod_{j<k}\left|\lambda_{j}-\lambda_{k}\right|^{\beta} \times \prod_{k=1}^{n} \lambda_{k}^{\frac{\beta}{2}(\kappa-n+1)-1} e^{-\frac{\beta}{2} \lambda_{k}}
$$

When $\kappa$ is an integer and $\beta=1$ or 2 this is the joint law of Laguerre eigenvalues just described (granting $\kappa \geq n$ which can be assumed with no loss of generality by the obvious duality). Notice though that the above law is sensible for any real $\kappa>n-1$ and $\beta>0$. As an application of our universal limit theorem, we also show:

Theorem 1.4. Let $\lambda_{1} \geq \lambda_{2} \geq \ldots$ denote the ordered $\beta$-Laguerre "eigenvalues" (1.9), and set

$$
\mu_{n, \kappa}=(\sqrt{n}+\sqrt{\kappa})^{2} \quad \text { and } \quad \sigma_{n, \kappa}=\frac{(\sqrt{n \kappa})^{1 / 3}}{(\sqrt{n}+\sqrt{\kappa})^{4 / 3}} .
$$

Then for any $k$, as $n \rightarrow \infty$ with arbitrary $\kappa=\kappa_{n}>n-1$ we have

$$
\left(\sigma_{n, \kappa}\left(\mu_{n, \kappa}-\lambda_{\ell}\right)\right)_{\ell=1, \ldots, k} \Rightarrow\left(\Lambda_{0}, \Lambda_{1}, \ldots, \Lambda_{k-1}\right) .
$$

In summary, we have a fairly complete characterization of the general beta random matrix "soft-edge". Within the context of log-gas type ensembles, the present is the first paper to rigorously establish local fluctuation theorems of any type away from the classical $\beta=1,2$ or 4 exponents on the Vandermonde interaction component of the density. Since its appearance, there have been several further advances in the general beta picture: 24] and 32] provide descriptions of the bulk spectrum, with [27] considering the so-called "hard-edge". The second and third papers rely in part on the results here, and based on 32 those authors have gone on to prove a refined form of Dyson's conjectures for the bulk eigenvalue spacing for all $\beta>0$ [33.

Once again, Section 2 discusses the definition and basic properties of $\mathrm{SAE}_{\beta}$. The diffusion connection and Theorem 1.2 are detailed in Section 3. Section 4 establishes the tail bounds, Theorem 1.3. In Section 5 we prove a general result, Theorem [5.1] which provides weak conditions under which the lowest eigenvalues of tridiagonal random matrices of type discrete Laplacian plus potential converge to the corresponding eigenvalues of the continuum operator limit. It is anticipated that a result of this type will be of future importance in investigations of universality in random matrix theory, and in Section 6 it is employed to prove Theorems 1.1 and 1.4 . 


\section{Basic properties of the stochastic Airy equation}

Definition of the stochastic Airy operator. We use the usual Schwartz distribution theory. Recall that the space of distributions $D=D\left(\mathbb{R}^{+}\right)$is the continuous dual of the space $C_{0}^{\infty}$ of all smooth compactly supported test functions under the topology of uniform-on-compact convergence of all derivatives. Recall as well that all continuous functions $f$ and their formal derivatives are distributions. They act on $C_{0}^{\infty}$ via integration by parts,

$$
\prec \varphi, f^{(k)} \succ:=(-1)^{k} \int f(x) \varphi^{(k)}(x) d x,
$$

where the latter is clearly defined. For instance, $b^{\prime}$, the formal derivative of Brownian motion, is a random distribution, as $b$ is a random continuous function. The notation $\prec \cdot, \cdot \succ$ distinguishes the above from an $L^{2}$ inner product $\langle\cdot, \cdot\rangle$.

Introduce $H_{\text {loc }}^{1}$, the space of functions $f: \mathbb{R}^{+} \rightarrow \mathbb{R}$ for which $f^{\prime} \mathbb{1}_{I} \in L^{2}$ for any compact set $I . \mathrm{SAE}_{\beta}$ is then well defined as a random linear map $H_{\text {loc }}^{1} \rightarrow D$, sending $f$ to the distribution

$$
\mathcal{H}_{\beta} f=-f^{\prime \prime}+x f+\frac{2}{\sqrt{\beta}} f b^{\prime} .
$$

As $D$ is only closed under multiplication by smooth functions, one must make sense of $f b^{\prime}$ as an element of that space. Stieltjes integration by parts prompts $\int_{0}^{y} f b^{\prime} d x:=-\int_{0}^{y} b f^{\prime} d x+f(y) b_{y}-f(0) b_{0}$. The latter is a continuous function of $y$, and we define $f b^{\prime}$ as its derivative.

Eigenvalues and eigenfunctions. We will consider the eigenvalues from two points of view:

(i) as the solutions of $\mathcal{H}_{\beta} f=\lambda f$ with given "boundary conditions", or

(ii) as solutions of the usual problem.

The first approach is intimately tied to the Riccati transformation, which will be our main tool for analyzing solutions. The second will be useful in obtaining bounds on the eigenvalue distributions for $\mathrm{SAE}_{\beta}$. Their equivalence for $\mathrm{SAE}_{\beta}$ is established over the course of the next two subsections.

Recall the Hilbert space $L^{*}$ defined via the norm

$$
\|f\|_{*}^{2}=\int_{0}^{\infty}\left(\left(f^{\prime}\right)^{2}+(1+x) f^{2}\right) d x, \quad L^{*}=\left\{f: f(0)=0,\|f\|_{*}<\infty\right\} .
$$

The framework just introduced makes it self-evident how to define the $L^{*}$ eigenfunctions and eigenvalues of $\mathrm{SAE}_{\beta}$ in the sense of (i).

Definition 2.1. The eigenvalues and eigenfunctions of $\mathcal{H}_{\beta}$ are the pairs $(f, \lambda) \in$ $L^{*} \times \mathbb{R}$ satisfying

$$
\mathcal{H}_{\beta} f=\lambda f,
$$

where both sides are interpreted as distributions $\left(L^{*} \subset C^{0}\left(\mathbb{R}^{+}\right) \subset D\right.$, where $C^{0}\left(\mathbb{R}^{+}\right)$ are those continuous functions on the half-line, vanishing at infinity). The $(k+1)$ st smallest point in this set, if it exists, will be denoted $\Lambda_{k}$.

The $f \in L^{*}$ requirement allows us to avoid technicalities. Proposition 3.7 shows that it can be relaxed to $f \in C^{0} \cap L^{2}$, or even further.

If we rewrite $\mathcal{H}_{\beta} f=\lambda f$ as

$$
f^{\prime \prime}=\left(x-\lambda+\frac{2}{\sqrt{\beta}} b^{\prime}\right) f,
$$


the right-hand side is a derivative of a continuous function, and thus $f^{\prime}$ can be taken to be continuous (it is defined in an a.e. sense). In this way we arrive at two equivalent formulations of the eigenvalue problem. One is the coincidence of the integrated versions of both sides of (2.1). The other is the equivalence of the two sides as distributions. The first reproduces the definition (1.5) from the introduction,

$$
f^{\prime}(x)-f^{\prime}(0)=\int_{0}^{x}\left[(y-\lambda) f-\frac{2}{\sqrt{\beta}} b_{y} f^{\prime}(y)\right] d y+\frac{2}{\sqrt{\beta}} f(x) b_{x},
$$

showing at once that $f^{\prime}$ inherits the Hölder $(1 / 2)^{-}$continuity properties of $b$, i.e., that it is $\operatorname{Hölder}(1 / 2-\varepsilon)$ continuous for all $\varepsilon>0$. Thus $f \in \operatorname{Hölder}(3 / 2)^{-}$. The second, weak definition, will be useful in the variational analysis. It reads

$$
\int \varphi^{\prime \prime} f d x=\int(x-\lambda) \varphi f d x+\int \frac{2}{\sqrt{\beta}}\left[\int_{0}^{x} b_{y} f^{\prime}(y) d y-b_{x} f(x)\right] \varphi^{\prime} d x
$$

and takes the form

$$
\int \varphi^{\prime \prime} f d x=\int(x-\lambda) f \varphi-\frac{2}{\sqrt{\beta}} b f^{\prime} \varphi-\frac{2}{\sqrt{\beta}} b f \varphi^{\prime} d x
$$

after an integration by parts.

Before proceeding to the variational approach, we register some simple facts about $L^{*}$.

Fact 2.2. Any $L^{*}$ bounded sequence has a subsequence $f_{n}$ that converges to some $f \in L^{*}$ in each of the following ways: (i) $f_{n} \rightarrow_{L^{2}} f$, (ii) $f_{n}^{\prime} \rightarrow f^{\prime}$ weakly in $L^{2}$, (iii) $f_{n} \rightarrow f$ uniformly-on-compacts and (iv) $f_{n} \rightarrow f$ weakly in $L^{*}$.

Proof. Convergence modes (ii) and (iv) are simple applications of the BanachAlaoglu theorem. (iii) stems from the familiar estimates $f_{n}^{2}(x) \leq 2\left\|f_{n}\right\|_{2}\left\|f_{n}^{\prime}\right\|_{2}$ and $\left|f_{n}(y)-f_{n}(x)\right|=\left|\int f_{n}^{\prime} \mathbb{1}_{[x, y]} d z\right| \leq\left\|f_{n}^{\prime}\right\|_{2}|x-y|^{1 / 2}$, showing that the sequence is uniformly equicontinuous on compacts. Last, (iii) implies $L^{2}$ convergence locally, while the bound $\sup _{n} \int x f_{n}^{2} d x<\infty$ produces the uniform integrability required for (i).

The quadratic form $\prec f, \mathcal{H}_{\beta} f \succ$. The quadratic form $\prec f, \mathcal{H}_{\beta} f \succ$ typically associated with selfadjoint operators is already defined for test functions $f \in C_{0}^{\infty}$. As a path to generalization, we proceed by decomposing the fluctuation term via

$$
b=\bar{b}+(b-\bar{b}), \quad \bar{b}_{x}=\int_{x}^{x+1} b_{y} d y, \quad \text { and so } \quad \bar{b}_{x}^{\prime}=b_{x+1}-b_{x} .
$$

The idea is to smooth out the noise and then later control the difference between the noise and its mollification. We have: for any $f \in C_{0}^{\infty}$,

$$
\prec f^{2}, b^{\prime} \succ=\int_{0}^{\infty} f^{2}(x) \bar{b}_{x}^{\prime} d x+2 \int_{0}^{\infty} f^{\prime}(x) f(x)\left(\bar{b}_{x}-b_{x}\right) d x .
$$

The necessary extension requires this object to be finite over $L^{*}$. Step one is a simple bound on the Brownian paths. 
Lemma 2.3. For $b_{x}, x>0$ a Brownian motion, there is a random constant $C<\infty$ so that

$$
\sup _{x>0} \sup _{0<y \leq 1} \frac{\left|b_{x+y}-b_{x}\right|}{\sqrt{\log (2+x)}} \leq C \quad \text { a.s. }
$$

As a consequence, $\left|\bar{b}^{\prime}(x)\right| \vee\left|\bar{b}_{x}-b_{x}\right| \leq C \sqrt{\log (2+x)}$.

Proof. By the triangle inequality $\left|b_{x+y}-b_{x}\right| \leq\left|b_{x+y}-b_{\lfloor x+y\rfloor}\right|+\left|b_{\lfloor x+y\rfloor}-b_{\lfloor x\rfloor}\right|+$ $\left|b_{x}-b_{\lfloor x\rfloor}\right|$ it is enough to show that

$$
\sup _{n>1} \frac{X_{n}}{\sqrt{\log n}} \leq C
$$

where the $X_{n}=\sup _{0<y<1}\left|b_{n+y}-b_{n}\right|$ are independent and identically distributed. Further, $P\left(X_{n}>a\right)=2 \sqrt{2 / \pi} \int_{a}^{\infty} e^{-m^{2} / 2} d m \leq 2 a^{-1} e^{-a^{2} / 2}$, courtesy of the reflection principle. A Borel-Cantelli argument completes the proof.

Using the lemma, we may certainly bound $\left|\bar{b}_{x}^{\prime}\right|$ by $C(1+x)$ and $\left|\bar{b}_{x}-b_{x}\right|$ by $\sqrt{C(1+x)}$. An application of Cauchy-Schwarz in (2.4) then yields

Proposition 2.4. There is the bound $\left|\prec f^{2}, b^{\prime} \succ\right| \leq C\|f\|_{*}^{2}$ for $C=C(b)<\infty$ a.s. In particular, $\prec f, \mathcal{H}_{\beta} f \succ$ is defined for all $f \in L^{*} ; \prec f, \mathcal{H}_{\beta} g \succ$ can be defined by polarization and is a continuous symmetric bilinear form $\left(L^{*}\right)^{2} \rightarrow \mathbb{R}$.

Variational characterization. We are at last in position to define the ground state energy $\tilde{\Lambda}_{0}=\tilde{\Lambda}_{0}(\beta, \omega)$ of $\mathcal{H}_{\beta}$ variationally. As anticipated, we will show that $\tilde{\Lambda}_{0}=\Lambda_{0}$, the lowest point in the set of eigenvalues of $\mathcal{H}_{\beta}$. Setting

$$
\tilde{\Lambda}_{0}:=\inf \left\{\prec f, \mathcal{H}_{\beta} f \succ: f \in L^{*} \text { with } f(0)=0,\|f\|_{2}=1\right\},
$$

we begin by proving that $\tilde{\Lambda}_{0}>C_{2}(\omega)>-\infty$ a.s. From now on, random constants are denoted by $C$., and deterministic ones by $c$.

Lemma 2.5. There are constants $c_{1}, C_{2}, C_{3}$ so that a.s. for all $f \in L^{*}$,

$$
c_{1}\|f\|_{*}^{2}-C_{2}\|f\|_{2}^{2} \leq \prec f, \mathcal{H}_{\beta} f \succ \leq C_{3}\|f\|_{*}^{2} .
$$

Proof. The upper bound is from Proposition 2.4. For the lower bound, we repeat the definition:

$$
\begin{aligned}
\prec f, \mathcal{H}_{\beta} f \succ= & \int_{0}^{\infty}\left(f^{\prime}\right)^{2} d x+\int_{0}^{\infty} x f^{2} d x \\
& +\frac{2}{\sqrt{\beta}} \int_{0}^{\infty} f^{2}(x) \bar{b}_{x}^{\prime} d x+\frac{4}{\sqrt{\beta}} \int_{0}^{\infty} f^{\prime}(x) f(x)\left(\bar{b}_{x}-b_{x}\right) d x .
\end{aligned}
$$

The first line on the right amounts to $\|f\|_{*}^{2}-\|f\|_{2}^{2}$, and it suffices to show that the terms $B_{1}, B_{2}$ in the second line do not ruin the picture and satisfy $B_{1}+B_{2} \geq$ $-c_{1}\|f\|_{*}^{2}-C\|f\|_{2}^{2}$ with $c_{1}<1$.

Lemma 2.3 provides the bounds $\frac{2}{\sqrt{\beta}}\left|\bar{b}_{x}^{\prime}\right| \leq c_{2}(C+x)$ and $\frac{2}{\sqrt{\beta}}\left|\bar{b}_{x}-b_{x}\right| \leq c_{2} \sqrt{C+x}$ for arbitrarily small $c_{2}$ and some random $C=C\left(c_{2}, b\right)$. Thus, $\left|B_{1}\right| \leq c_{2}\|f\|_{*}^{2}+$ $c_{2} C\|f\|_{2}^{2}$, and for the second term we have

$$
\left|B_{2}\right| \leq \int_{0}^{\infty}\left|f^{\prime}(x) f(x)\right| c_{2} \sqrt{C+x} d x \leq c_{2}\left\|f^{\prime}(x)\right\|_{2}^{2}+c_{2} \int_{0}^{\infty}(C+x) f^{2}(x) d x .
$$

In particular, $|B| \leq 3 c_{2}\|f\|_{*}^{2}+C^{\prime}\|f\|_{2}^{2}$, where again $c_{2}$ may be taken as small as needed. 
Corollary 2.6. The infimum in the variational problem (2.6) is attained at an eigenfunction $f_{0}$ of $\mathcal{H}_{\beta}$ with eigenvalue $\Lambda_{0}$.

Proof. Again by Lemma 2.3, for any $\varepsilon>0$ the bounds $\left|\bar{b}_{x}^{\prime}\right| \leq \varepsilon(1+x)$ and $\left|b_{x}-b_{x}\right| \leq$ $\varepsilon \sqrt{1+x}$ hold for all $x>X(b, c)$. As in the proof of Lemma 2.5 it follows that

$\prec f, \mathcal{H}_{\beta} f \succ=\|f\|_{*}^{2}-\|f\|_{2}^{2}+\frac{2}{\sqrt{\beta}} \int_{0}^{X} f^{2}(x) \bar{b}_{x}^{\prime} d x+\frac{4}{\sqrt{\beta}} \int_{0}^{X} f^{\prime}(x) f(x)\left(\bar{b}_{x}-b_{x}\right) d x+\mathcal{E}$,

where the error term satisfies $|\mathcal{E}| \leq \varepsilon\|f\|_{*}^{2}$.

Next choose a minimizing sequence $f_{n} \in L^{*}:\left\|f_{n}\right\|_{2}=1$ with $\prec f_{n}, \mathcal{H}_{\beta} f_{n} \succ \rightarrow \tilde{\Lambda}_{0}$. By Lemma 2.5. $\left\|f_{n}\right\|_{*}<B$ for some $B=B(b)$, and by Fact 2.2, we can find a subsequence along which $f_{n} \rightarrow f_{0}$ uniformly on compacts, in $L^{2}$, and also weakly in $H^{1}$. Terms 2, 3, 4 on the right-hand side of (2.8) then converge to their evaluations at $f$, while term 1 plainly satisfies $\|f\|_{*}^{2} \leq \liminf \left\|f_{n}\right\|_{*}^{2}$. Letting $\varepsilon \rightarrow 0$ yields $\prec f_{0}, \mathcal{H}_{\beta} f_{0} \succ \leq \tilde{\Lambda}_{0}$, with the opposite inequality holding by definition.

To complete the picture, taking the functional derivative of the variational problem (2.6) in directions $\varphi \in C_{0}^{\infty}$, that is $\frac{d}{d \varepsilon} \prec f_{0}+\varepsilon \varphi,\left.\mathcal{H}_{\beta}\left(f_{0}+\varepsilon \varphi\right) \succ\right|_{\varepsilon=0}$, shows that the selected minimizer satisfies $\mathcal{H}_{\beta} f=\tilde{\Lambda}_{0} f_{0}$ in the sense of distributions. It is therefore an eigenfunction and $\tilde{\Lambda}_{0}=\Lambda_{0}$.

The above formulation may now be used to define higher eigenvalues in the expected manner: $\tilde{\Lambda}_{1}$ arising from restricting the class of potential minimizers to be perpendicular to $f_{0}$, and so on. This defines a sequence of eigenvalues $\tilde{\Lambda}_{k}, k \geq 0$, each of which is finite. That $\cdots<\tilde{\Lambda}_{k}<\tilde{\Lambda}_{k+1}<\cdots$ is proved later; see Proposition 3.5. The standard trick with the bilinear form defined in Lemma 2.4 yields the $L^{2}$-orthogonality of the corresponding eigenfunctions (and, as a by-product, the uniqueness of the ground state found above). In short:

Lemma 2.7. The $(k+1)$ st lowest element $\Lambda_{k}$ in the set of eigenvalues of $\mathcal{H}_{\beta}$ exists and in fact $\Lambda_{k}=\tilde{\Lambda}_{k}$.

Remark 2.8. Lemma2.3. as used in Lemma2.5, is related to the second condition of our general convergence result, Theorem 5.1 , below. In addition, a discrete version of this estimate is employed in the context of the $\beta$-ensembles converging to $\mathrm{SAE}_{\beta}$.

\section{RICCATI TRANSFORM AND DIFFUSION FORMULAS}

The Riccati map is a classical tool in the study one-dimensional random Schrödinger spectra. Its use dates back to Halperin [21] who computed the density of states for the operator $-\frac{d^{2}}{d x^{2}}+b_{x}^{\prime}$. For applications to local statistics such as the ground state energy, see [5], 6], and [25].

Return to the eigenvalue problem (1.5),

$$
\psi^{\prime \prime}(x)=\frac{2}{\sqrt{\beta}} \psi(x) b_{x}^{\prime}+(x-\lambda) \psi(x),
$$

understood in the integration-by-parts sense. The Riccati transform is simply the logarithmic derivative $p(x)=\psi^{\prime}(x) / \psi(x)$. This turns (3.1) into a first-order differential equation: $p(0)=\infty$, and

$$
p^{\prime}(x)=x-\lambda-p^{2}(x)-\frac{2}{\sqrt{\beta}} b^{\prime}(x),
$$

understood in the same way. 
Solutions of (3.2) may blow up (to $-\infty)$ at finite times, as will happen whenever $\psi$ vanishes. In this case $p$ is immediately restarted at $+\infty$ at that time-point in order to continue the solution corresponding to (3.1). It is convenient to think of $p$ as taking values in the disjoint union of countable copies of the reals, $\mathbb{R}_{0}, \mathbb{R}_{-1}, \mathbb{R}_{-2}, \ldots$.. Points $(n, x)$ in this space are ordered lexicographically, though we sometimes refer to these points by their second coordinate $x$. A natural topology on this space is provided by the two-point compactification of each copy of the reals, glued together at the endpoints so as to respect the lexicographic ordering. (This ordering and topology can also be defined by considering the evolution of $\arg \left(\psi^{\prime}+i \psi\right)$ as a continuous, real-valued function, and applying the tangent map.)

It is not hard to verify the following.

Fact 3.1. The solution $p_{\lambda}(x)=p(x, \lambda)$ of (3.2) is unique and increasing in $\lambda$ for each $x$. It is also decreasing in $x$ at each blowup (or "explosion"). Moreover, the function $p$ is continuous when the image space is considered in the topology discussed above.

Next consider the truncation $\mathcal{H}^{L}$ of $\mathcal{H}_{\beta}$, defined on the finite interval $[0, L]$ with Dirichlet $(\psi=0)$ boundary conditions at both endpoints.

Lemma 3.2. Fix $\lambda$, and denote $(-n, y)=p(L, \lambda)$. Then the number $n$ of blowups of $p(x, \lambda)$ to $-\infty$ on $[0, L]$ equals the number of eigenvalues of $\mathcal{H}^{L}$ at most $\lambda$.

Proof. We provide just a sketch. First, $\lambda$ is an eigenvalue of $\mathcal{H}^{L}$ if and only if $p_{\lambda}$ blows up to $-\infty$ at the endpoint $L$. For large negative $\lambda$, there are no blowups for any given noise path. As $\lambda$ increases, continuity and monotonicity imply that existing blowups move towards the beginning of the interval; new ones can only appear at the endpoint $L$. At those $\lambda$ we have a new eigenvalue and the claim follows.

To extend the picture to the full line we need the following.

Lemma 3.3. As $L \rightarrow \infty$ the first $k$ eigenvalues $\Lambda_{L, 0}, \ldots, \Lambda_{L, k-1}$ of $\mathcal{H}^{L}$ converge to the first $k$ eigenvalues of $\mathcal{H}_{\beta}$.

Proof. A trivial modification of the proof of Lemma 5.9 together with Fact 2.2 shows that $\lim \inf \Lambda_{L, k} \geq \Lambda_{k}$. Next, for an inductive proof, assume that the $\Lambda_{L, \ell} \rightarrow \Lambda_{\ell}$ for $\ell<k$.

Let $f_{k}^{\varepsilon}$ be a function of compact support $\varepsilon$-close to $f_{k}$ in $L^{*}$. Also let

$$
g_{L}=g_{L, k}=f_{k}^{\varepsilon}-\sum_{\ell=0}^{k}\left\langle f_{k}^{\varepsilon}, f_{L, \ell}\right\rangle f_{L, \ell} .
$$

The hypothesis entails that $f_{L, \ell} \rightarrow_{L^{2}} f_{\ell}$. So, for large $L$, each coefficient in the above sum is bounded by $2 \varepsilon$, and $g_{L}$ will be $c \varepsilon$-close to $f_{k}$ in $L^{*}$. Then, by the variational characterization we have

$$
\limsup _{L \rightarrow \infty} \Lambda_{L, k} \leq \limsup _{L \rightarrow \infty} \frac{\prec g_{L}, \mathcal{H}_{\beta} g_{L} \succ}{\left\langle g_{L}, g_{L}\right\rangle},
$$

since $f_{k}^{\varepsilon}$ is eventually supported on $[0, L]$. Then, as $\varepsilon \rightarrow 0$, the right-hand side converges to $\prec f_{k}, \mathcal{H}_{\beta} f_{k} \succ /\left\langle f_{k}, f_{k}\right\rangle=\Lambda_{k}$. 
Taking the $L \rightarrow \infty$ limit of the claims of Lemma 3.2 then yields

Proposition 3.4. Let $N(\lambda)$ be the number of blowups of the equation (3.2) to $-\infty$. Then for almost all $\lambda, N(\lambda)$ equals the number of eigenvalues of $\mathcal{H}_{\beta}$ at most $\lambda$. In other words, the cadlag version of $N(\lambda)$ is $\mathcal{H}_{\beta}$ 's eigenvalue counting function.

Of course, for any fixed $\lambda$, the Riccati equation may be taken in the Itô sense,

$$
d p(x)=-\frac{2}{\sqrt{\beta}} d b_{x}+\left(x-\lambda-p^{2}(x)\right) d x,
$$

which is to say that $p=p_{\lambda}=(\log \psi)^{\prime}$ performs the indicated diffusion, restarted at $+\infty$ instantaneously after each explosion to $-\infty$. The content of the above is that the total explosion count equals the count of eigenvalues $\leq \lambda$.

Proof of Theorem 1.2. The strong Markov property of the motion (3.3) implies that the sequence of explosion times, $\mathfrak{m}_{0}=0, \mathfrak{m}_{1}, \mathfrak{m}_{2}, \ldots$ is itself a Markov process. Let $\kappa(x, \cdot)$ be the distribution of the first such time of $p_{0}(x)$ under $\mathbb{P}_{(\infty, x)}$, that is, when started from $\infty$ at time $x$. This law is supported on $(x, \infty]$ with a point mass at $\infty$. By the preceding,

$$
\begin{aligned}
\mathbb{P}\left(\Lambda_{k-1}<\lambda\right) & =\mathbb{P}_{(\infty, 0)}\left(p_{\lambda}(x) \text { has at least } k \text { explosions }\right) \\
& =\mathbb{P}_{(\infty,-\lambda)}\left(p_{0}(x) \text { has at least } k \text { explosions }\right) \\
& =\int_{\mathbb{R}^{k}} \kappa\left(-\lambda, d x_{1}\right) \kappa\left(x_{1}, d x_{2}\right) \ldots \kappa\left(x_{k-1}, d x_{k}\right) .
\end{aligned}
$$

The second equality uses the obvious translation equivariance of $p$.

In addition:

Proposition 3.5. The $\mathcal{H}_{\beta}$ eigenvalues are a.s. distinct with no accumulation point. In particular, $\Lambda_{k} \rightarrow \infty$ as $k \rightarrow \infty$ a.s., and the minimization procedure of the previous section exhausts the full $\mathcal{H}_{\beta}$ eigenvalue set.

Proof. The distinctness follows from the fact that $\mathbb{P}(N(\lambda+)-N(\lambda) \leq 1)=1$. Next, starting at any time $x$, there is positive probability $\kappa(x,\{\infty\})$ that $p_{0}$ will converge to $\infty$ without blowing up to $-\infty$. Monotonicity implies that $\kappa(x,\{\infty\})$ is increasing in $x$. Thus the number of eigenvalues below $\lambda$ is dominated by a geometric random variable with parameter $\kappa(-\lambda,\{\infty\})$, whence it is finite. The claim follows.

We mention that the second half of Proposition 3.5 may also be established by making sense of the resolvent operator of $\mathcal{H}_{\beta}$ and showing it maps the unit ball in $L^{*}$ onto Hölder $(3 / 2)^{-}$functions vanishing at infinity. The simplicity of the above proof demonstrates the advantages of the diffusion picture.

This connection between the limiting top eigenvalues of the random matrix ensembles and the explosion time of a simple, one-dimensional diffusion is new even in the deeply studied cases of $\beta=1,2$ or 4 . We now recall the formulas of TracyWidom which, in conjunction with our result, produce the identities

$\mathbb{P}_{(\infty, \lambda)}\left(\mathfrak{m}_{1}=\infty\right)= \begin{cases}\exp \left(-\frac{1}{2} \int_{\lambda}^{\infty}(s-\lambda) u^{2}(s) d s\right) \exp \left(-\frac{1}{2} \int_{\lambda}^{\infty} u(s) d s\right), & \beta=1, \\ \exp \left(-\int_{\lambda}^{\infty}(s-\lambda) u^{2}(s) d s\right), & \beta=2, \\ \exp \left(-\frac{1}{2} \int_{\lambda^{\prime}}^{\infty}\left(s-\lambda^{\prime}\right) u^{2}(s) d s\right) \cosh \left(\frac{1}{2} \int_{\lambda^{\prime}}^{\infty} u(s) d s\right), & \beta=4 .\end{cases}$

Here, $u(s)$ is the solution of $u^{\prime \prime}=s u+2 u^{3}$ (Painlevé II) subject to $u(s) \sim \operatorname{Ai}(s)$ as $s \rightarrow+\infty$, and $\lambda^{\prime}=2^{2 / 3} \lambda$ in the $\beta=4$ distribution. An important problem for the 
future is to obtain the equivalent of the closed Tracy-Widom formulas for general $\beta$. Even a direct verification of the above $\beta=1,2,4$ formuals would be interesting.

Remark 3.6. The diffusion (3.3) seems efficient for simulating Tracy-Widom distributions as well as distributions of higher eigenvalues. First note that for $x \ll 1$, $p(x)$ comes down from $+\infty$ like $1 / x$. Also, the more time accumulated inside the parabola $\rho_{\lambda}=\left\{p^{2}(x) \leq x+\lambda\right\}$, the less likely explosion becomes. That is, the typical path which hits $-\infty$ does so by tunneling through the narrow part of $\rho_{\lambda}$. In line with these heuristics good simulations of the general $\mathrm{TW}_{\beta}$ distributions may be obtained by tracking the explosion probability for $p(x)$ begun at say $p(0)=O\left(10^{3}\right)$ and run only for $O(1)$ time.

Moving to applications of the Riccati map to the eigenfunctions, a detailed but standard analysis of the diffusion (3.3) using domination arguments shows that for each $\lambda$, with probability one, $p(x) / \sqrt{x} \rightarrow 1$ (after a finite number of initial blowups and restarts). By Fubini, this holds for almost all $\lambda$. By monotonicity, this happens for all $\lambda$ except for eigenvalues. Thus we get

Proposition 3.7. A.s. for all solutions $f \in H_{l o c}^{1}$ of $\mathcal{H}_{\beta} f=\lambda f$, with $f(0)=0$ we have the following. If $f$ is not an eigenfunction, then $f^{\prime}(x) /(f(x) \sqrt{x}) \rightarrow 1$. In particular, if $f$ grows slower than $\exp \left((2 / 3-\varepsilon) x^{3 / 2}\right)$, then $f$ is an eigenfunction.

Remark 3.8. Note that the results of this section, and indeed, the entire paper, easily extend to general initial boundary conditions.

We conclude this section with a decay bound on the $\mathcal{H}_{\beta}$ eigenfunctions. Compare this to the noiseless $(\beta=\infty)$ limit in which case all eigenfunctions are simply shifts of the Airy function $\mathrm{Ai}(\cdot)$.

Proposition 3.9. Let $f$ be any eigenfunction of $\mathcal{H}_{\beta}$. Then, for any $\varepsilon>0$ there is a random constant $C_{f, \varepsilon}$ so that

$$
|f(x)| \leq C_{f, \varepsilon} \exp \left(-(2 / 3-\varepsilon) x^{3 / 2}\right)
$$

with probability one.

Proof. Let $p, q$ be the solutions of (3.2) corresponding to Dirichlet and Neumann boundary conditions at 0 (i.e. $q(0)=0$ ), with $p=f^{\prime} / f$ for the specified eigenfunction $f$. Then $q$ cannot correspond to an eigenfunction of the Neumann problem and so by Proposition 3.7 (in conjunction with Remark 3.8) $q(x) / \sqrt{x} \rightarrow 1$. From the differential equation (3.2) we have

$$
\frac{d}{d x}(q-p)=-(q-p)(q+p)
$$

and so

$$
(q-p)(x)=C \exp \left(\int_{\mathfrak{m}}^{x}-(q+p)(y) d y\right)
$$

for $\mathfrak{m}<\infty$ some random time past the final explosion of $q$ with $p(\mathfrak{m})$ finite. With the notation $Q(x)=\int_{\mathfrak{m}}^{x} q(y) d y, P(x)=\int_{\mathfrak{m}}^{x} p(y) d y$, and $R(x)=Q(x)-P(x)$ the above reads

$$
R^{\prime}(x)=C \exp (R(x)-2 Q(x)) .
$$

Now $Q(x)=(2 / 3) x^{3 / 2}(1+o(1))$ and $P(x)=C+\log |f(x)| \rightarrow-\infty$, implying that

$$
R(x)-x^{3 / 2} \rightarrow \infty .
$$


To finish, it suffices to show that for all $\varepsilon>0$ and $x>x_{0}(\varepsilon)$ we have $R(x) \geq$ $(4 / 3)(1-\varepsilon) x^{3 / 2}$. Assume the contrary. Then we can find $x_{0}$ large so that the right-hand side of (3.5) at $x=x_{0}$ is at most 1 and for $x>x_{0}$ we have $q(x)>1 / 2$. We claim that the solutions of the ODE (3.5) started at $x=x_{0}$ are dominated by the solutions of $\hat{R}^{\prime}(x)=1$. Indeed, when $R(x) \leq \hat{R}(x)$,

$$
\begin{aligned}
C \exp (R(x)-2 Q(x)) & \leq C \exp (\hat{R}(x)-2 Q(x)) \\
& =C \exp \left(R\left(x_{0}\right)-2 Q\left(x_{0}\right)+\int_{x_{0}}^{x} 1-2 q(y) d y\right) \leq 1
\end{aligned}
$$

so that the monotonicity can be maintained. Thus $R(x) \leq \hat{R}(x)=C+x$ for all large $x$, contradicting (3.6).

\section{Tracy-Widom tail Bounds: An application of $\mathrm{SAE}_{\beta}$}

This section contains the proof of Theorem 1.3 .

With $T W_{\beta}=-\Lambda_{0}(\beta)$ now defined by (2.6), both the upper bound on $\mathbb{P}\left(T W_{\beta}<\right.$ $-a)$ and the lower bound on $P\left(T W_{\beta}>a\right)$ follow from suitable choices of the test function $f$ in

$$
\prec f, \mathcal{H}_{\beta} f \succ=\int_{0}^{\infty}\left[f^{\prime}(x)^{2}+x f^{2}(x)\right] d x+\frac{2}{\sqrt{\beta}} \int_{0}^{\infty} f^{2}(x) d b_{x} \geq \Lambda_{0}\|f\|_{2}^{2} .
$$

The other two bounds run through the Riccati correspondence.

Lower bound, right tail. Begin with the observation that

$$
\begin{aligned}
\mathbb{P}\left(T W_{\beta}>a\right) & =\mathbb{P}\left(\Lambda_{0}(\beta)<-a\right) \\
& \geq \mathbb{P}\left(\prec f, \mathcal{H}_{\beta} f \succ<-a\langle f, f\rangle\right) \\
& =\mathbb{P}\left(\frac{2}{\sqrt{\beta}}\|f\|_{4}^{2} \mathfrak{g}<-a\|f\|_{2}^{2}-\left\|f^{\prime}\right\|_{2}^{2}-\|\sqrt{x} f\|_{2}^{2}\right)
\end{aligned}
$$

for any choice of $f \in L^{*}$, and $\mathfrak{g}$ a standard Gaussian variable. Here we have used the rule that for $h$ deterministic, $\int_{0}^{\infty} h d b$ is a centered Gaussian with variance $\|h\|_{2}^{2}$.

Wishing to maximize this probability, the observation is: for there to be a large negative eigenvalue the random potential, and then so also the eigenfunction $f$, should be localized. This leads one to neglect the $\int_{0}^{\infty} x f^{2}$ term and look for the maximizer $f$ of the expression entering the Gaussian tail, namely

$$
\frac{\int_{0}^{\infty}\left(a f^{2}+f^{\prime 2}\right)}{\left(\int_{0}^{\infty} f^{4}\right)^{1 / 2}}
$$

Viewing this problem on the whole line and neglecting boundary conditions, the optimizers can be computed exactly: $f(x)=c_{1} \operatorname{sech}\left(\sqrt{a} x+c_{2}\right)$.

Note that $\int_{-\infty}^{\infty} \operatorname{sech}^{2}(x) d x=2, \int_{-\infty}^{\infty}\left(\operatorname{sech}^{\prime}(x)\right)^{2} d x=2 / 3$, and $\int_{-\infty}^{\infty} \operatorname{sech}^{4}(x) d x=$ $4 / 3$. Let $f(x)=\operatorname{sech}(\sqrt{a}(x-1))$. Then, on $\mathbb{R}^{+}$, with $\sim$ denoting asymptotics as $a \uparrow \infty$ we have

$$
a\|f\|_{2}^{2} \sim 2 \sqrt{a}, \quad\left\|f^{\prime}\right\|_{2}^{2} \sim \frac{2}{3} \sqrt{a}, \quad\|\sqrt{x} f\|_{2}^{2}=O\left(\frac{1}{\sqrt{a}}\right), \quad\|f\|_{4}^{4} \sim \frac{4}{3 \sqrt{a}} .
$$

Further, while $f(0) \neq 0$, it decays exponentially there as $a \rightarrow \infty$, allowing for an admissible modification which shares the above evaluations. Returning to (4.1) we 
find that

$$
\mathbb{P}\left(T W_{\beta}>a\right) \geq \mathbb{P}\left(\frac{2}{\sqrt{\beta}} \times \frac{2}{\sqrt{3}} a^{-1 / 4} \mathfrak{g}<-a^{1 / 2}\left(2+\frac{2}{3}+o(1)\right)\right),
$$

producing the desired bound from the simple Gaussian tail estimate $\mathbb{P}(\mathfrak{g}>c)=$ $e^{-c^{2}(1 / 2+o(1))}$.

Upper bound, left tail. The reasoning is the same as that just employed, though in minimizing the right-hand side of $\mathbb{P}\left(T W_{\beta}<-a\right) \leq \mathbb{P}\left(\prec f, \mathcal{H}_{\beta} f \succ>a\langle f, f\rangle\right)$ one expects the optimal $f$ to be relatively "flat". Neglecting the $\int\left(f^{\prime}\right)^{2}$ term leads to the choice

$$
f(x)=(x \sqrt{a}) \wedge \sqrt{(a-x)^{+}} \wedge(a-x)^{+} .
$$

The middle term is dominant, while the others control $\left\|f^{\prime}\right\|_{2}$. Then

$$
a\|f\|_{2}^{2} \sim \frac{a^{3}}{2}, \quad\left\|f^{\prime}\right\|_{2}^{2}=O(a), \quad\|\sqrt{x} f\|_{2}^{2} \sim \frac{a^{3}}{6}, \quad\|f\|_{4}^{4} \sim \frac{a^{3}}{3} .
$$

The proof is completed by substitution:

$$
\begin{aligned}
\mathbb{P}\left(T W_{\beta}<-a\right) & \leq \mathbb{P}\left(\frac{2}{\sqrt{\beta}} \times \frac{1}{\sqrt{3}} a^{3 / 2} \mathfrak{g}>a^{3}\left(\frac{1}{2}-\frac{1}{6}+o(1)\right)\right) \\
& =\exp \left(-\frac{\beta}{24} a^{3}(1+o(1))\right) .
\end{aligned}
$$

Lower bound, left tail. For this we use the diffusion description of Theorem 1.2 , namely $\mathbb{P}\left(T W_{\beta}<-a\right)=\mathbb{P}_{(\infty,-a)}(p$ never explodes), where $p$ is the diffusion (1.8), and the subscript indicates the space-time starting point. By monotonicity,

$$
\begin{aligned}
& \mathbb{P}_{(\infty,-a)}(p \text { never explodes }) \geq \mathbb{P}_{(1,-a)}(p \text { never explodes }) \\
& \quad \geq \mathbb{P}_{(0,-a)}(p(x) \in[0,2] \text { for all } x \in[-a, 0]) \mathbb{P}_{(0,0)}(p \text { never explodes }) .
\end{aligned}
$$

The last factor in line two is some positive number not depending on $a$. To bound the first probability from below, employ the Cameron-Martin-Girsanov formula

$$
\begin{aligned}
& \mathbb{P}_{(1,-a)}(p(x) \in[0,2] \text { for all } x \in[-a, 0]) \\
& =\mathbb{E}_{(1,-a)}\left[\exp \left(-\frac{\beta}{4} \int_{-a}^{0}\left(x-b_{x}^{2}\right) d b_{x}-\frac{\beta}{8} \int_{-a}^{0}\left(x-b_{x}^{2}\right)^{2} d x\right) ;\right. \\
& \left.\qquad b_{x} \in[0,2] \text { for all } x \leq 0\right],
\end{aligned}
$$

where $b_{x}$ is a Brownian motion with diffusion coefficient $2 / \sqrt{\beta}$. On the event in question,

$$
\frac{\beta}{8} \int_{-a}^{0}\left(x-b_{x}^{2}\right)^{2} d x=\frac{\beta}{24} a^{3}+O\left(a^{2}\right)
$$

and

$$
\int_{-a}^{0}\left(x-b_{x}^{2}\right) d b_{x}=a b_{-a}+\frac{1}{3}\left(b_{-a}^{3}-b_{0}^{3}\right)+\left(\frac{4}{\beta}-1\right) \int_{-a}^{0} b_{x} d x=O(a) .
$$

To finish, note that $\mathbb{P}_{(-a, 0)}\left(b_{x} \in[0,2]\right.$ for $\left.x \leq 0\right) \geq e^{-c a}$ and so does not interfere with the leading order. 
Upper bound, right tail. We thank Laure Dumaz for helping correct an earlier, flawed attempt. The present proof is based on her master's thesis [13, which contains more precise estimates. Write, for $a \gg 1$ and a large $c>0$ to be determined,

$$
\mathbb{P}\left(T W_{\beta}>a\right)=\mathbb{P}_{\infty}\left(\mathfrak{m}_{-\infty}<\infty\right) \leq \mathbb{P}_{\sqrt{a}-c}\left(\mathfrak{m}_{-\sqrt{a}}<\infty\right),
$$

where $\mathfrak{m}$ denotes the time passage to the indicated level of the process

$$
d p(x)=\frac{2}{\sqrt{\beta}} d b_{x}+\left(a+x-p^{2}(x)\right) d x .
$$

To bound the rightmost probability in (4.2) we introduce a further control on the paths, and show that there is a numerical constant $c^{\prime}$ so that

$$
\mathbb{P}_{\sqrt{a}-1}\left(\mathfrak{m}_{-\sqrt{a}}<\infty\right) \leq c^{\prime} \mathbb{P}_{\sqrt{a}-1}\left(\mathfrak{m}_{-\sqrt{a}}<\infty, \mathfrak{m}_{\sqrt{a}}>\mathfrak{m}_{-\sqrt{a}}\right) .
$$

This is accomplished by two applications of the (strong) Markov property. From now on we denote $\mathfrak{m}_{ \pm}=\mathfrak{m}_{ \pm \sqrt{a}}$ and $\mathcal{A}=\left\{\mathfrak{m}_{+}>\mathfrak{m}_{-}\right\}$and have

$$
\begin{aligned}
\mathbb{P}_{\sqrt{a}-c}\left(\mathfrak{m}_{-}<\infty\right) & =\mathbb{P}_{\sqrt{a}-c}\left(\mathfrak{m}_{-}<\infty, \mathcal{A}\right)+\mathbb{P}_{\sqrt{a}-c}\left(\mathfrak{m}_{-}<\infty, \mathcal{A}^{c}\right) \\
& \leq \mathbb{P}_{\sqrt{a}-c}\left(\mathfrak{m}_{-}<\infty, \mathcal{A}\right)+\mathbb{P}_{\sqrt{a}}\left(\mathfrak{m}_{-}<\infty\right) \\
& \leq \mathbb{P}_{\sqrt{a}-c}\left(\mathfrak{m}_{-}<\infty, \mathcal{A}\right)+\mathbb{P}_{\sqrt{a}}\left(\mathfrak{m}_{\sqrt{a}-c}<\infty\right) \mathbb{P}_{\sqrt{a}-c}\left(\mathfrak{m}_{-}<\infty\right) .
\end{aligned}
$$

Both inequalities use the fact that the hitting probability of any level below the starting place is decreasing in the starting time of the diffusion $p$. The desired bound (4.3) then lies in the following claim, the proof of which we defer to the end.

Claim 4.1. There exists a large enough $c$ so that $\mathbb{P}_{\sqrt{a}}\left(\mathfrak{m}_{\sqrt{a}-c}=\infty\right)$ is uniformly bounded below (i.e., independently of $a \gg c$ ).

We proceed by performing a change of measure,

$$
\mathbb{P}_{\sqrt{a}-c}\left(\mathfrak{m}_{-}<\infty, \mathcal{A}\right)=\lim _{L \rightarrow \infty} \mathbb{E}_{\sqrt{a}-c}\left[R(q, L), \mathfrak{m}_{+}<\mathfrak{m}_{-}<L\right],
$$

where $q$ is the diffusion with reversed drift,

$$
d q(x)=\frac{2}{\sqrt{\beta}} d b(x)+\left(q^{2}(x)-x-a\right) d x,
$$

and the Cameron-Martin-Girsanov factor is given by

$$
\log R(q, L)=\frac{\beta}{2} \int_{0}^{L \wedge \mathfrak{m}_{-}}\left(a+x-q^{2}(x)\right) d q(x) .
$$

An application of Itô's lemma shows that, for any $z>0$,

$$
\begin{aligned}
\int_{0}^{z}\left(a+x-q^{2}(x)\right) d q(x)= & a(q(z)-q(0))-\frac{1}{3}\left(q^{3}(z)-q^{3}(0)\right) \\
& +z q(z)+(4 / \beta-1) \int_{0}^{z} q(x) d x .
\end{aligned}
$$

For $z \leq \mathfrak{m}_{-} \wedge \mathfrak{m}_{+}$, then $|q(x)| \leq \sqrt{a}$ for $x \in[0, z]$, so the first line is bounded by a function of $a$, and the second line is bounded by $(1+|4 / \beta-1|) \sqrt{a} \mathfrak{m}_{-}=c_{1} \sqrt{a} \mathfrak{m}_{-}$. We will show that

$$
\log \mathbb{E}_{\sqrt{a}-c}\left[e^{c_{1} \sqrt{a} \overline{\mathfrak{m}}}, \mathfrak{m}_{+}<\mathfrak{m}_{-}\right]=o\left(a^{3 / 2}\right)
$$


Letting $L \rightarrow \infty$ in (4.4), the dominated convergence theorem combined with (4.5) gives

$$
\mathbb{P}\left(T W_{\beta}>a\right) \leq c^{\prime} e^{-\frac{2}{3} \beta a^{3 / 2}(1+o(1))},
$$

since for $z=\mathfrak{m}_{-}$and $q(0)=\sqrt{a}-c$ the first line of (4.5) equals $-(4 / 3) a^{3 / 2}+O(a)$.

Next recall that the expectation (4.6) is in terms of the $q$-diffusion, and notice that on the event $\left\{\mathfrak{m}_{+}<\mathfrak{m}_{-}\right\}$the $q$-drift is bounded above by $-x$. Therefore, if we introduce the process $\bar{q}(x)=\frac{2}{\sqrt{\beta}} b(x)-x^{2} / 2$ and let $\overline{\mathfrak{m}}=\inf \{x>0: \bar{q}(x)=-\sqrt{a}\}$, it is enough to show that $\log \mathbb{E}_{\sqrt{a}}\left[e^{c \sqrt{a} \overline{\mathfrak{m}}}\right]=o\left(a^{3 / 2}\right)$ for any constant $c$. For this, the simple bound

$$
\mathbb{P}_{\sqrt{a}}(\overline{\mathfrak{m}}>t) \leq \mathbb{P}_{\sqrt{a}}\left(\frac{2}{\sqrt{\beta}} b(t)>t^{2}-\sqrt{a}\right) \leq e^{-\frac{\beta}{8} t^{3}} \text { for } t>4\left(1+\frac{2}{\sqrt{\beta}}\right) \sqrt{a}
$$

will do the job.

To finish, we return to the proof behind the key bound (4.3).

Proof of Claim 4.1. Certainly, the probability that $p$, begun at $\sqrt{a}$, never reaches $\sqrt{a}-c$ is bounded below by the same probability for $p$ replaced by its reflected (downward) at $\sqrt{a}$ version. Further, when restricted to the interval $[\sqrt{a}-c, \sqrt{a}]$, the $p$-diffusion has drift everywhere bounded below by $x$. Thus we may consider instead the same probability for the appropriate reflected Brownian motion with quadratic drift.

To formalize this, it is convenient to shift orientation. Now let $\bar{p}(x)=\frac{2}{\sqrt{\beta}} b(x)-$ $x^{2} / 2$, and let $p^{*}(x)$ denote $\bar{p}$ reflected (upward) at the origin. Namely,

$$
p^{*}(x)=\bar{p}(x)-\inf _{y<x} \bar{p}(y) .
$$

If we can show that for a large enough $c, \mathbb{P}_{0}\left(p^{*}(x)\right.$ never reaches $\left.c\right)>0$, then the $p$-probability in question will also be bounded below, independent of $a$.

What we actually prove is that $M=\sup _{x>0} p^{*}(x)$ is a.s. finite. Let $\kappa>1$, and for each $n \geq 0$ define the event

$$
D_{n}=\left\{\bar{p}(x) \text { hits }(1-n) \kappa \text { for some } x \text { between } \mathfrak{m}_{-n \kappa} \text { and } \mathfrak{m}_{-(n+1) \kappa}\right\} .
$$

From the representation (4.8) one sees that $M \geq 2 \kappa$ implies that some $D_{n}$ must occur. Define as well the event

$$
A_{\kappa}=\left\{\bar{p}(x) \geq-\frac{1}{2}\left(\kappa x^{2}+1\right) \text { for all } x>0\right\},
$$

and note that, on $A_{\kappa}, \mathfrak{m}_{-n \kappa} \geq \sqrt{2 n-1}$. Hence, still on $A_{\kappa}$, for all $n \geq 1$ the shifted process $\bar{p}\left(\mathfrak{m}_{-n \kappa}+x\right)-\bar{p}\left(\mathfrak{m}_{-n \kappa}\right)$ is dominated by the scaled Brownian motion $x \mapsto \frac{2}{\sqrt{\beta}} b(x)$ plus the constant drift $-(1 / 2) \sqrt{2 n-1}$. It follows that: for $n \geq 1$,

$$
\begin{aligned}
\mathbb{P} & \left(D_{n} \cap A_{\kappa} \mid \mathcal{F}_{\mathfrak{m}_{-n \kappa}}\right) \\
& \leq \mathbb{P}_{0}\left(x \mapsto\left(\frac{2}{\sqrt{\beta}} b(x)-\frac{1}{2} \sqrt{2 n-1} x\right) \text { hits } \kappa \text { before }-\kappa\right) \\
& =\frac{1}{1+e^{\kappa(\beta / 2) \sqrt{2 n-1}}} .
\end{aligned}
$$


Putting the above together we have

$$
\begin{aligned}
\mathbb{P}(M>2 \kappa) & \leq \mathbb{P}\left(A_{\kappa}^{c}\right)+\mathbb{P}\left(A_{\kappa} \cap\left(\bigcup_{n \geq 0} D_{n}\right)\right) \\
& \leq \mathbb{P}\left(A_{\kappa}^{c}\right)+\mathbb{P}\left(D_{0}\right)+\sum_{n \geq 1} \mathbb{P}\left(A_{\kappa} \cap D_{n}\right) .
\end{aligned}
$$

The sum of the series converges to 0 by (4.9). Since $D_{0}$ implies that $\sup _{x>0} \bar{p}(x)>$ $\kappa$, both $\mathbb{P}\left(A_{\kappa}^{c}\right)$ and $\mathbb{P}\left(D_{0}\right)$ tend to zero as $\kappa \rightarrow \infty$ by the Law of the Iterated Logarithm.

\section{Convergence of Discrete models And Universality}

This section establishes a general and rather weak set of conditions under which the bottom eigenvalues of random symmetric tridiagonal matrices converge to the bottom eigenvalues of a corresponding stochastic differential operator. In many ways this is the central result of the paper.

To explain the setup, consider a sequence of discrete-time $\mathbb{R}^{2}$-valued random sequences $\left(\left(y_{n, 1, k}, y_{n, 2, k}\right) ; 1 \leq k \leq n\right)$. Let $m_{n}=o(n)$ be a scaling parameter. (In the particular case of the Hermite $\beta$-ensembles we have $m_{n}=n^{1 / 3}$.) For each $n$, we build an $n \times n$ tridiagonal matrix $H_{n}$.

Let $T_{n}$ denote the shift operator $\left(T_{n} v\right)_{k}=v_{k+1}$ acting on $\mathbb{R}_{1} \times \mathbb{R}_{2} \times \ldots$ Let $\left(T_{n}^{t} v\right)_{k}=v_{k-1} 1_{k \geq 1}$ be its adjoint, and let $R_{n}$ denote the restriction operator $\left(R_{n} v\right)_{k}=v_{k} \mathbb{1}_{k \leq n}$. Also let $\Delta_{n}=m_{n}\left(I-T_{n}^{t}\right)$ be the difference quotient operator, and finally set

$$
H_{n}=R_{n}\left(-\Delta_{n} \Delta_{n}^{t}+\left(\Delta_{n} y_{n, 1}\right)_{\times}+\frac{1}{2}\left(\left(\Delta_{n} y_{n, 2}\right)_{\times} T_{n}+T_{n}^{t}\left(\Delta_{n} y_{n, 2}\right)_{\times}\right)\right),
$$

where the subscript $\times$ denotes element-wise multiplication by the corresponding vector. Then $H_{n}$ maps the coordinate subspace $\mathbb{R}^{n} \rightarrow \mathbb{R}^{n}$, and its matrix with respect to the coordinate basis in $\mathbb{R}^{n}$ is symmetric tridiagonal with $\left(2 m_{n}^{2}+m_{n}\left(y_{n, 1, k}-\right.\right.$ $\left.\left.y_{n, 1, k-1}\right), k \geq 1\right)$ on the diagonal and $\left(-m_{n}^{2}+m_{n}\left(y_{n, 2, k}-y_{n, 2, k-1}\right) / 2, k \geq 1\right)$ below and above the diagonal. Roughly speaking, $H_{n}$ is the discrete Laplacian plus integrated potential $y_{n, 1}+y_{n, 2}$.

Additionally, define $y_{n, i}(x)=y_{n, i,\left\lfloor x m_{n}\right\rfloor} 1_{x m_{n} \in[0, n]}$. By choice, $\left(\sqrt{m_{n}} \times y_{n, i, k}, k \geq\right.$ 0 ) is on the scale of a simple random walk, so no additional spatial scaling will be required.

Our basic convergence result rests on two sets of assumptions on the processes $y_{n, i=1,2}$.

Assumption 1 (Tightness/Convergence). There exists a continuous process $x \mapsto$ $y(x)$ such that

$$
\begin{aligned}
& \left(y_{n, i}(x) ; x \geq 0\right) \quad i=1,2 \quad \text { are tight in law, } \\
& \left(y_{n, 1}(x)+y_{n, 2}(x) ; x \geq 0\right) \Rightarrow(y(x) ; x \geq 0) \quad \text { in law, }
\end{aligned}
$$

with respect to the Skorokhod topology of paths; see 18 for the definitions.

Assumption 2 (Growth/Oscillation bound). There is a decomposition

$$
y_{n, i, k}=m_{n}^{-1} \sum_{\ell=1}^{k} \eta_{n, i, \ell}+w_{n, i, k}
$$


for $\eta_{n, i, k} \geq 0$, deterministic, unbounded nondecreasing continuous functions $\bar{\eta}(x)>$ $0, \zeta(x) \geq 1$, and random constants $\kappa_{n}(\omega) \geq 1$ defined on the same probability space which satisfy the following. The $\kappa_{n}$ are tight in distribution, and, almost surely,

$$
\begin{aligned}
\bar{\eta}(x) / \kappa_{n}-\kappa_{n} \leq \eta_{n, 1}(x)+\eta_{n, 2}(x) & \leq \kappa_{n}(1+\bar{\eta}(x)), \\
\eta_{n, 2}(x) & \leq 2 m_{n}^{2}, \\
\left|w_{n, 1}(\xi)-w_{n, 1}(x)\right|^{2}+\left|w_{n, 2}(\xi)-w_{n, 2}(x)\right|^{2} & \leq \kappa_{n}(1+\bar{\eta}(x) / \zeta(x))
\end{aligned}
$$

for all $n$ and $x, \xi \in\left[0, n / m_{n}\right]$ with $|x-\xi| \leq 1$.

We may now define the limiting operator. Just as in Section 2, we note that

$$
H=-\frac{d^{2}}{d x^{2}}+y^{\prime}(x)
$$

maps $H_{\mathrm{loc}}^{1}$ to the space $D$ of distributions via integration by parts. Without changing the notation, we generalize the Hilbert space $L^{*} \subset L^{2}\left(\mathbb{R}^{+}\right)$introduced there. This consists of functions with $f(0)=0$ and

$$
\|f\|_{*}^{2}=\int_{0}^{\infty}\left[f^{\prime}(x)^{2}+(1+\bar{\eta}(x)) f^{2}(x)\right] d x<\infty .
$$

The eigenvalues and eigenfunctions are defined again as $(\lambda, f) \in \mathbb{R} \times L^{*} \backslash\{0\}$ with $\|f\|_{2}=1$ satisfying (5.7). Recall from Section 2 that this means that

$$
f^{\prime}(x)=\int_{0}^{x}\left(-y(z) f^{\prime}(z)-\lambda f\right) d z+f(x) y(x),
$$

or, equivalently, for all $\varphi \in C_{0}^{\infty}$ it follows that

$$
\int f \varphi^{\prime \prime} d x=\int\left(-\lambda f \varphi-y f^{\prime} \varphi-y f \varphi^{\prime}\right) d x
$$

With the picture laid out, a few words on Assumptions 1 and 2 are in order. The former simply asks for the correspondence between $H_{n}$ and $H$ at the level of integrated potentials. The latter, more technical condition, will imply the compactness necessary to maintain a discrete spectrum as $n \uparrow \infty$.

Theorem 5.1 (Convergence in law). Given Assumptions 1 and 2 above and any fixed $k$, the bottom $k$ eigenvalues of the matrices $H_{n}$ converge in law to the bottom $k$ eigenvalues of the operator $H$.

We will also show that, after a natural embedding, the eigenfunctions also converge in $L^{2}$.

Proof: Reduction to the deterministic case. It will be convenient to find subsequences along which we have limits for all desired quantities.

The upper bound (5.4) shows that $\left(\int_{0}^{x} \eta_{n, i}(t) d t ; x \geq 0\right)$ is tight in distribution for $i=1,2$. For any subsequence we can extract a further subsequence so that we have joint distributional convergence

$$
\begin{aligned}
\left(\int_{0}^{x} \eta_{n, i}(t) d t ; x \geq 0\right) & \Rightarrow\left(\eta_{i}^{\dagger}(t) d t ; x \geq 0\right), \\
\left(y_{n, i}(x) ; x \geq 0\right) & \Rightarrow\left(y_{i}(x) ; x \geq 0\right), \\
\kappa_{n} & \Rightarrow \kappa
\end{aligned}
$$

where the convergence in the first lines is in the uniform-on-compacts topology, and the second, in the Skorokhod topology. Then by Skorokhod's representation 
theorem (see Theorem 1.8, Chapter 2 of [18]) we can realize this convergence as a.s. convergence on some probability space so that the conditions of Proposition 5.2 below are satisfied with probability one.

Note that (5.4)-(5.5) are local Lipschitz bounds on the $\int \eta_{n, i}$, and so they are inherited by their limit $\eta_{i}^{\dagger}$. Thus $\eta_{i}=\left(\eta_{i}^{\dagger}\right)^{\prime}$ is defined almost everywhere, and satisfies (5.4)-(5.5). Further, $\eta_{i}$ can be defined everywhere so that (5.4)-(5.5) continues to hold.

It also follows that each $w_{n, i}=y_{n, i}-\sum \eta_{n, i}$ must have a limit, which we denote by $w_{i}$. We further denote $w=w_{1}+w_{2}$ and $\eta=\eta_{1}+\eta_{2}$. The claim now follows from Proposition 5.2 below.

Proposition 5.2 (Deterministic convergence). Assume that each of the convergence statements in (5.8) holds deterministically and that the bounds (5.4)-(5.6) hold with some deterministic constant $\kappa$ replacing $\kappa_{n}$. Then, for any $k$, the lowest $k$ eigenvalues of the matrices $H_{n}$ converge to the lowest $k$ eigenvalues of $H$.

In the next subsection, we establish some properties of the limiting operator. Afterwards, we prove Proposition 5.2 .

Properties of the limiting operator. Just as in Section 2, we extend the bilinear form $\prec \cdot, H \cdot \succ$ from $C_{0}^{\infty} \times L^{*}$ to $L^{*} \times L^{*}$. We want to define the extension as $\prec f, H f \succ:=\int\left(f^{\prime 2}+\eta f^{2}\right) d x+\int f^{2} d w$ (with $\prec f, H g \succ$ then defined by polarization), but we still need to define and control the last term. By the next lemma, this can be done via the integration by parts already employed in (2.4):

$\int_{0}^{\infty} f^{2}(x) d w_{x}=\int_{0}^{\infty} f^{2}(x)\left(w_{x+1}-w_{x}\right) d x+2 \int_{0}^{\infty} f^{\prime}(x) f(x)\left(\int_{x}^{x+1} w_{t} d t-w_{x}\right) d x$.

Lemma 5.3. The integrals on the right of (5.9) are defined and finite for $f \in L^{*}$. Moreover there exist $c_{8}, c_{9}, c_{10}>0$ so that

$$
c_{8}\|f\|_{*}^{2}-c_{9}\|f\|_{2}^{2} \leq \prec f, H f \succ \leq c_{10}\|f\|_{*}^{2} .
$$

Proof. By taking limits of the inequalities (5.4)-(5.6) on $\eta_{i, n}$ and $w_{n}$ we get bounds for $\eta_{i}$ and $w$. In particular, $\max \left(\left|w_{x+1}-w_{x}\right|,\left|w_{x+1}-w_{x}\right|^{2}\right) \leq c_{\varepsilon}+\varepsilon \bar{\eta}$, where $\varepsilon$ can be made small. Now we write $\prec f, H f \succ=A+B$, where $B$ is the fluctuation term (5.9), and the potential term satisfies $\frac{1}{\kappa}\|f\|_{*}^{2}-c\|f\|_{2}^{2} \leq A \leq c\|f\|_{*}^{2}$. To bound $B$, first write

$$
\left.\int_{0}^{\infty} f^{2}(x)\left|w_{x+1}-w_{x}\right| d x \leq\left\langle f,\left(c_{\varepsilon}+\varepsilon\right) \bar{\eta}\right) f\right\rangle
$$

For the second term, we average the inequality $\sup _{|x-\xi| \leq 1}\left|w_{\xi}-w_{x}\right| \leq\left|c_{\varepsilon}+\varepsilon \bar{\eta}(x)\right|^{1 / 2}$ and use an inequality of means:

$$
2 \int_{0}^{\infty}\left|f^{\prime}(x) f(x)\left(\int_{x}^{x+1} w_{t} d t-w_{x}\right)\right| d x \leq \sqrt{\varepsilon}\left\|f^{\prime}\right\|_{2}^{2}+\left\langle f, \frac{1}{\sqrt{\varepsilon}}\left(c_{\varepsilon}+\varepsilon \bar{\eta}\right) f\right\rangle .
$$

The above inequalities give $|B| \leq 2 \sqrt{\varepsilon}\|f\|_{*}^{2}+c_{\varepsilon}^{\prime}\|f\|_{2}^{2}$. Setting $\varepsilon$ small we get the results.

The bounds immediately imply the following.

Corollary 5.4. (i) The bilinear form $\prec \cdot, H \cdot \succ: L^{*} \times L^{*} \rightarrow \mathbb{R}$ is continuous. (ii) It does not depend on the decomposition $y=w+\int \eta$. (iii) The eigenvalues and 
eigenfunctions $(\lambda, f)$ of $H$ satisfy $\prec g, H f \succ=\lambda\langle g, f\rangle$ for all $g \in L^{*}$. (iv) In particular, $\prec f, H f \succ=\lambda\langle f, f\rangle$.

Proof of the corollary. Since $L^{*} \subset L^{2}$ is a continuous embedding, it suffices to prove that $\prec \cdot H \cdot \succ+c_{9}\langle\cdot, \cdot\rangle$ is continuous. This form is nonnegative definite by the lemma. Continuity (i) now follows from Cauchy-Schwarz applied to the form and the bounds of the lemma. For (ii) and (iii), approximate $g$ by smooth compactly supported functions and use continuity.

Together, these two statements provide an analogue of Lemma 2.5 in a more general context. In particular, the (discrete) eigenvalues of $H$ may now be defined variationally. The arguments used in Section 2 in conjunction with the limiting bounds of (5.4)-(5.6) give the following.

Lemma 5.5. The lowest $k$ elements of the set of eigenvalues of $H$ exist and admit the variational characterization via the bilinear form $\prec f, H f \succ$.

Tightness. Next, we define the discrete analogue of the norm $\|\cdot\|_{*}$. For $v \in \mathbb{R}^{n}$ let $\|v\|_{2}^{2}=m_{n}^{-1} \sum_{k=1}^{n} v_{k}^{2}$ with scaling to match the continuum norm. Let $\bar{\eta}_{n, k}=$ $\bar{\eta}\left(k / m_{n}\right)$ and let

$$
\|v\|_{* n}^{2}=\left\|\Delta_{n} v\right\|_{2}^{2}+\left\|\left(\bar{\eta}_{n, .}\right)^{1 / 2} v\right\|_{2}^{2}+\|v\|_{2}^{2},
$$

and note that $\|v\|_{2} \leq\|v\|_{* n}$. We continue with a bound on $H_{n}$.

Lemma 5.6. Assume the bounds (5.4)-(5.6). Then there are constants $c_{11}, c_{12}$, $c_{13}>0$ so that for all $n$ and all $v$ we have

$$
c_{11}\|v\|_{* n}^{2}-c_{12}\|v\|_{2}^{2} \leq\left\langle v, H_{n} v\right\rangle \leq c_{13}\|v\|_{* n}^{2} .
$$

Proof. Drop the subscript $n$ and recall the definition of the difference quotient $\Delta v_{k}=m\left(v_{k+1}-v_{k}\right)$. We recall the following consequence of the discretized bounds (5.4) $-(5.6)$ :

$$
\begin{aligned}
\bar{\eta}_{k} / \kappa-\kappa & \leq \eta_{1, k}+\eta_{2, k} \leq \kappa \bar{\eta}_{k}+\kappa, \\
\eta_{2, k} & \leq 2 m^{2}, \\
\left|w_{i, \ell}-w_{i, k}\right|^{2} & \leq \varepsilon \bar{\eta}_{k}+c_{\varepsilon}, \quad k \leq \ell \leq k+m, \quad i=1,2 .
\end{aligned}
$$

Here $\varepsilon$ can be arbitrarily small at the expense of $c_{\varepsilon}$. Let $w_{k}=w_{1, k}, u_{k}=w_{2, k}$. By definition of $H_{n}$,

$$
m\left\langle v, H_{n} v\right\rangle=\sum_{k=0}^{n}\left(\left(\Delta v_{k}\right)^{2}+\eta_{2, k} v_{k} v_{k+1}+\eta_{1, k} v_{k}^{2}\right)+\sum_{k=0}^{n}\left(\Delta w_{k} v_{k}^{2}+\Delta u_{k} v_{k} v_{k+1}\right) .
$$

Let $A, B$ denote the two sums. Using the inequality $a b \geq-(b-a)^{2} / 3+a^{2} / 4$, we obtain the following lower bound for the second summand in $A$ :

$$
\eta_{2, k} v_{k} v_{k+1} \geq-\frac{\eta_{2, k}}{3}\left(v_{k+1}-v_{k}\right)^{2}+\frac{\eta_{2, k} v_{k}^{2}}{4} \geq-\frac{2}{3}\left(\Delta v_{k}\right)^{2}+\frac{\bar{\eta}_{2, k} v_{k}^{2}}{4 \kappa}-\frac{\kappa v_{k}^{2}}{4}
$$


thus we get $A \geq \frac{m}{4 \kappa}\|v\|_{*}^{2}-c m\|v\|^{2}$. We also clearly have $|A| \leq c m\|v\|_{*}^{2}$. To bound $|B|$, set $\delta w_{k}=\left(w_{k+m}-w_{k}\right)$. Summation by parts gives

$$
\begin{aligned}
\sum_{k=0}^{n} \Delta w_{k} v_{k}^{2} & =\sum_{k=0}^{n}\left(\Delta w_{k}-\delta w_{k}\right) v_{k}^{2}+\sum_{k=0}^{n} \delta w_{k} v_{k}^{2} \\
& =\sum_{k=0}^{n}\left(\sum_{\ell=k+1}^{k+m}\left(w_{\ell}-w_{k}\right)\right)\left(v_{k+1}^{2}-v_{k}^{2}\right)+\sum_{k=0}^{n} \delta w_{k} v_{k}^{2} .
\end{aligned}
$$

By the bound on $w$ the absolute value of the first summand in (5.12) is not more than

$$
m\left(\varepsilon \bar{\eta}_{k}+c_{\varepsilon}\right)^{1 / 2}\left|v_{k+1}^{2}-v_{k}^{2}\right| \leq \frac{1}{\sqrt{\varepsilon}}\left(\varepsilon \bar{\eta}_{k}+c_{\varepsilon}\right)\left(v_{k}+v_{k+1}\right)^{2}+\sqrt{\varepsilon} m^{2}\left(v_{k+1}-v_{k}\right)^{2} .
$$

Together with the bound on the second sum in (5.12), this yields

$$
m\left|\sum_{k=0}^{n} \Delta w_{k} v_{k}^{2}\right| \leq\left(\frac{1}{\sqrt{\varepsilon}}+1\right) \sum_{k=0}^{n}\left(\varepsilon \bar{\eta}_{k}+c_{+} \varepsilon\right) v_{k}^{2}+\sqrt{\varepsilon} m^{2} \sum_{k=0}^{n}\left(v_{k+1}-v_{k}\right)^{2} .
$$

The argument for $u$ starts with the same summation by parts, with $v_{k} v_{k+1}$ playing the part of $v_{k}^{2}$. After bounding the $u$ terms, we use the inequalities $2\left|v_{k} v_{k+1}\right| \leq$ $v_{k}^{2}+v_{k+1}^{2}$ and

$$
\left|v_{k} v_{k-1}-v_{k} v_{k+1}\right| \leq\left|v_{k}\right|\left|v_{k}-v_{k-1}\right|+\left|v_{k}\right|\left|v_{k+1}-v_{k}\right|
$$

together with Cauchy-Schwarz to get an estimate of the form (5.13) for the term $\sum_{k=0}^{n} \Delta u_{k} v_{k} v_{k+1}$. Thus we find that $|B| \leq c \sqrt{\varepsilon} m\|v\|_{*}^{2}+c_{\varepsilon}^{\prime} m\|v\|_{2}^{2}$. For $\varepsilon$ sufficiently small, the claims follow.

Operator convergence. We embed the domain $\mathbb{R}^{n}$ of $H_{n}$ in $L^{2}\left(\mathbb{R}^{+}\right)$in an isometric way, identifying $v \in \mathbb{R}^{n}$ with the step function $v(x)=v_{\left\lceil m_{n} x\right\rceil}$ supported on $\left[0, n / m_{n}\right]$. Let $L_{n}^{*}$ denote the space of such step functions, and let $\mathcal{P}_{n}$ denote the $L^{2}$-projection to this space. Let $\left(T_{n} f\right)(x)=f\left(x+m_{n}^{-1}\right)$ denote the shift operator, and let $R_{n}(f)=f \mathbb{1}_{\left[0, n / m_{n}\right]}$ denote the restriction. Let $\Delta_{n}=m_{n}\left(I-T_{n}^{t}\right)$. These operators are simply extensions of the already defined action of $T_{n}$ and $\Delta_{n}$ on $L_{n}^{*}$. Thus the formula (5.1) extends the definition of $H_{n}$ to $L^{2}$.

It is easy to check the following: (i) $\mathcal{P}_{n}$ and $T_{n}$, and so $\Delta_{n}$ commute; (ii) for $f \in L^{2}$ we have $\mathcal{P}_{n} f \rightarrow f$ in $L^{2}$; (iii) when $f^{\prime} \in L^{2}$ and $f(0)=0$ we have $\Delta_{n} f \rightarrow f^{\prime}$ in $L^{2}$.

Lemma 5.7. Assume that $f_{n} \in L_{n}^{*}$ and $f_{n} \rightarrow f$ weakly in $L^{2}$ and $\Delta_{n} f_{n} \rightarrow f^{\prime}$ weakly in $L^{2}$. Then for all $\varphi \in C_{0}^{\infty}$ we have $\left\langle\varphi, H_{n} f_{n}\right\rangle \rightarrow \prec \varphi, H f \succ$. In particular,

$$
\left\langle\mathcal{P}_{n} \varphi, H_{n} \mathcal{P}_{n} \varphi\right\rangle=\left\langle\varphi, H_{n} \mathcal{P}_{n} \varphi\right\rangle \rightarrow \prec \varphi, H \varphi \succ .
$$

Proof. Because we are dealing with $\varphi$ of compact support, we may drop the restriction part $R_{n}$ for $H_{n}$. The convergence

$$
\left\langle\varphi, \Delta_{n} \Delta_{n}^{t} f\right\rangle=\left\langle\Delta_{n} \Delta_{n}^{t} \varphi, f\right\rangle \rightarrow\left\langle\varphi^{\prime \prime}, f\right\rangle=\prec \varphi, f^{\prime \prime} \succ
$$

is clear, so it remains to check the potential term. First note that if $I$ is a finite interval, and $g_{n} \rightarrow_{L^{2}} g$ and $h_{n} \rightarrow h$ is $L^{2}(I)$-bounded and converges weakly in $L^{2}(I)$, then

$$
\left\langle g_{n}, h_{n} \mathbb{1}_{I}\right\rangle \rightarrow\left\langle g, h \mathbb{1}_{I}\right\rangle .
$$


Let $I$ be a finite closed interval supporting $\Delta_{n} \varphi, \varphi^{\prime}$ and $\varphi$. The potential term is

$$
\left\langle\varphi,\left(\left(\Delta_{n} y_{n, 1}\right)_{\times}+\frac{1}{2}\left(\left(\Delta_{n} y_{n, 2}\right)_{\times} T_{n}+T_{n}^{t}\left(\Delta_{n} y_{n, 2}\right)_{\times}\right)\right) f\right\rangle .
$$

Setting $y_{n}=y_{n, 1}+y_{n, 2}$, we first approximate the right-hand side by

$$
\begin{aligned}
\left\langle\varphi,\left(\Delta_{n} y_{n}\right)_{\times} f_{n}\right\rangle & =\left\langle\Delta_{n}^{t}\left(\varphi f_{n}\right), y_{n}\right\rangle \\
& =\left\langle\varphi \Delta_{n}^{t} f_{n}+f_{n} \Delta_{n}^{t} \varphi+m_{n}^{-1} \Delta_{n}^{t} f_{n} \Delta_{n}^{t} \varphi, y_{n}\right\rangle \\
& =\left\langle\Delta_{n}^{t} f_{n}, \varphi y_{n}\right\rangle+\left\langle f_{n}, y_{n} \Delta_{n}^{t} \varphi\right\rangle+m_{n}^{-1}\left\langle\Delta_{n}^{t} f_{n}, y_{n} \Delta_{n}^{t} \varphi\right\rangle .
\end{aligned}
$$

The first two terms in the above converge to the desired limits by (5.15), and the last one converges to 0 because it is bounded without the extra scaling term. The error term in the above approximation comes as a sum of $T_{n}$ and $T_{n}^{t}$ terms; we consider twice the $T_{n}$ part:

$$
\begin{aligned}
\left|\left\langle\varphi,\left(\Delta_{n} y_{n, 2}\right)_{\times}\left(I-T_{n}\right) f_{n}\right\rangle\right| & =\left|\left\langle\varphi m_{n}^{-1} \Delta_{n} y_{n, 2}, \Delta_{n} f_{n}\right\rangle\right| \\
& \leq\left\|m_{n}^{-1} \Delta_{n} y_{n, 2} \mathbb{1}_{I}\right\|_{2}\left\|\Delta_{n} f_{n}\right\|_{2} \sup _{x \in \mathbb{R}}|\varphi(x)| .
\end{aligned}
$$

Now $m_{n}^{-1} \Delta_{n} y_{n, 2} \mathbb{1}_{I}$ is the restriction to $I$ of the difference $y_{n, 2}-T_{n} y_{n, 2}$, in which both terms converge to $y_{2}$ in the Skorokhod topology. In particular, they converge a.e., and since they are locally bounded, their difference converges locally in $L^{2}$ to 0 . This shows that (5.16) vanishes in the limit. We handle the $T_{n}$ term similarly.

Lemma 5.8. Recall the discrete $\|\cdot\|_{* n}$ norm from (5.10). Assume that $f_{n} \in L_{n}^{*}$, $\left\|f_{n}\right\|_{* n} \leq c$, and $\left\|f_{n}\right\|_{2}=1$. Then there exists $f \in L^{*}$ and a subsequence $n_{k}$ so that $f_{n_{k}} \rightarrow L_{L^{2}} f$ and for all $\varphi \in C_{0}^{\infty}$ we have $\left\langle\varphi, H_{n_{k}} f_{n_{k}}\right\rangle \rightarrow \prec \varphi, H f \succ$.

Proof. Since $f_{n}$ and $\Delta_{n} f_{n}$ are bounded in $L^{2}$, we can find a subsequence along which $f_{n} \rightarrow f \in L^{2}$ and $\Delta_{n} f_{n} \rightarrow \tilde{f} \in L^{2}$ weakly. Considering $\left\langle\Delta_{n} f_{n}, 1_{[0, t]}\right\rangle$ we get that $\int \tilde{f}=f$; that is, $f$ has a differentiable version and $\tilde{f}=f^{\prime}$. The bounded nature of the $\bar{\eta}$ terms in the $L_{n}^{*}$ norm gives sufficient tightness so that we have $f \in L^{*}$ and $f_{n} \rightarrow_{L^{2}} f$. The last part then follows from Lemma 5.7.

We break up the proof of Proposition 5.2 into two lemmas. Let $\left(\lambda_{n, k}, v_{n, k}\right), k \geq 0$ be the lowest eigenvalues and the embedded normalized eigenfunctions of $H_{n}$, and let $\left(\Lambda_{k}, f_{k}\right)$ be the same for $H$.

Lemma 5.9. For $k \geq 0$ we have $\underline{\lambda}_{k}=\liminf \lambda_{k, n} \geq \Lambda_{k}$.

Proof. Assume $\underline{\lambda}_{k}<\infty$. Since the eigenvalues of $H_{n}$ are uniformly bounded below, we can find a subsequence so that $\left(\lambda_{n, 1}, \ldots, \lambda_{n, k}\right) \rightarrow\left(\xi_{1}, \ldots, \xi_{k}=\underline{\lambda}_{k}\right)$. By Lemma 5.6. the corresponding eigenfunctions have $L_{n}^{*}$ norm uniformly bounded, and Lemma 5.8 now implies that for a further subsequence, their $L^{2}$ limit exists. Moreover, by the same lemma this limit must consist of orthonormal eigenfunctions of $H$ with eigenvalues at most $\underline{\lambda}_{k}$. The orthonormality of the eigenfunction set shows that they correspond to $k$ distinct states and the proof is finished.

Lemma 5.10. For $k \geq 0$ we have $\lambda_{k, n} \rightarrow \Lambda_{k}$ and $v_{n, k} \rightarrow_{L^{2}} f_{k}$.

Proof. For an inductive proof, we assume the claim holds up to $k-1$. First, we find $f_{k}^{\varepsilon} \in C_{0}^{\infty} \varepsilon$-close to $f_{k}$ in $L^{*}$. Consider the vector

$$
f_{n, k}=\mathcal{P}_{n} f_{k}^{\varepsilon}-\sum_{\ell=1}^{k-1}\left\langle v_{n, \ell}, \mathcal{P}_{n} f_{k}^{\varepsilon}\right\rangle v_{n, \ell} .
$$


We have a uniform bound on the $L_{n}^{*}$ norm of $v_{n, \ell}$ by Lemma[5.6 and $\left|\left\langle v_{n, \ell}, \mathcal{P}_{n} f_{k}^{\varepsilon}\right\rangle\right| \leq$ $\left\|f_{k}^{\varepsilon}-f_{k}\right\|_{2}+\left\|v_{n, \ell}-f_{\ell}\right\|_{2}$, which, for large $n$, is bounded by $2 \varepsilon$. Thus the $L_{n}^{*}$ norm of the sum in (5.17) is bounded by $c \varepsilon$. By the uniform bound $\left\langle v, H_{n} v\right\rangle \leq c\|v\|_{* n}^{2}$ of Lemma 5.6 and the variational characterization in finite dimensions we also have that

$$
\begin{aligned}
\limsup \lambda_{n, k} & \leq \limsup _{n \rightarrow \infty} \frac{\left\langle f_{n, k}, H_{n} f_{n, k}\right\rangle}{\left\langle f_{n, k}, f_{n, k}\right\rangle} \\
& =\limsup _{n \rightarrow \infty} \frac{\left\langle\mathcal{P}_{n} f_{k}^{\varepsilon}, H_{n} \mathcal{P}_{n} f_{k}^{\varepsilon}\right\rangle}{\left\langle\mathcal{P}_{n} f_{k}^{\varepsilon}, \mathcal{P}_{n} f_{k}^{\varepsilon}\right\rangle}+o_{\varepsilon}(1),
\end{aligned}
$$

where $o_{\varepsilon}(1) \rightarrow 0$ as $\varepsilon \rightarrow 0$. Then (5.14) of Lemma 5.7 provides

$$
\lim _{n \rightarrow \infty}\left\langle\mathcal{P}_{n} f_{k}^{\varepsilon}, H_{n} \mathcal{P}_{n} f_{k}^{\varepsilon}\right\rangle=\prec f_{k}^{\varepsilon}, H f_{k}^{\varepsilon} \succ
$$

and therefore the right-hand side of (5.18) equals

$$
\frac{\prec f_{k}^{\varepsilon}, H f_{k}^{\varepsilon} \succ}{\left\langle f_{k}^{\varepsilon}, f_{k}^{\varepsilon}\right\rangle}+o_{\varepsilon}(1)=\frac{\prec f_{k}, H f_{k} \succ}{\left\langle f_{k}, f_{k}\right\rangle}+o_{\varepsilon}(1) .
$$

Now letting $\varepsilon \rightarrow 0$ the right-hand side converges to $\prec f_{k}, H f_{k} \succ /\left\langle f_{k}, f_{k}\right\rangle=\Lambda_{k}$. We have shown that $\lambda_{n, k} \rightarrow \Lambda_{k}$.

Lemma 5.8 implies that any subsequence of the $v_{n, k}$ has a further subsequence converging in $L^{2}$ to some $g \in L^{*}$ satisfying $H g=\Lambda_{k} g$. Thus $g=f_{k}$, and so $v_{n, k} \rightarrow_{L^{2}} f_{k}$.

\section{CLT AND tightness FOR TRIDiagonal $\beta$-Ensembles}

At last we verify that the $\beta$-Hermite and Laguerre ensembles satisfy the conditions (5.2)-(5.6) of Theorem [5.1] and so complete the proofs of Theorems 1.1 and 1.4.

The following theorem is what we need from the far more general Theorem 7.4.1 on page 354 in Ethier and Kurtz [18]. Denote $\triangle y_{n, k}=y_{n, k}-y_{n, k-1}$.

Corollary 6.1. Let $a \in \mathbb{R}$ and $h \in C_{1}\left(\mathbb{R}^{+}\right)$, and let $y_{n}$ be a sequence of processes with $y_{n, 0}=0$ and independent increments. Assume that

$$
\begin{aligned}
m_{n} \mathbb{E} \triangle y_{n, k} & =h^{\prime}\left(k / m_{n}\right)+o(1), \\
m_{n} \mathbb{E}\left(\triangle y_{n, k}\right)^{2} & =a^{2}+o(1), \\
m_{n} \mathbb{E}\left(\triangle y_{n, k}\right)^{4} & =o(1),
\end{aligned}
$$

uniformly for $k / m_{n}$ on compact sets as $n \rightarrow \infty$. Then $y_{n}(t)=y_{n,\left\lfloor t m_{n}\right\rfloor \text { converges }}$ in law, with respect to the Skorokhod topology, to the process $h(t)+a b_{t}$, where $b$ is standard Brownian motion.

Proof. The time-homogeneity required in the theorem can be replaced by introducing a space coordinate recording time. The supremum increment bound of the theorem follows from Markov's inequality and the fourth moment bound here. 
The $\beta$-Hermite case. Starting with the scaled Hermite matrix ensembles $H_{n}=$ $\tilde{H}_{n}^{\beta}$, we identify $m_{n}=n^{1 / 3}$. After rearranging some terms we find

$$
\begin{aligned}
& y_{n, 1, k}=w_{n, 1, k}=-n^{-1 / 6}(2 / \beta)^{1 / 2} \sum_{\ell=1}^{k} g_{\ell}, \\
& y_{n, 2, k}=n^{-1 / 6} \sum_{\ell=1}^{k} 2\left(\sqrt{n}-\frac{1}{\sqrt{\beta}} \chi_{\beta(n-\ell)}\right) .
\end{aligned}
$$

Also, by choosing $\eta_{n, 2, k}=2 \sqrt{n}-2 \beta^{-1 / 2} \mathbb{E} \chi_{\beta(n-k)}$, both $w_{n, 1, k}$ and $w_{n, 2, k}$ are independent-increment martingales. Using the notation and results of Corollary 6.1 and standard moment computations for the normal and gamma distributions, we get the following.

Lemma 6.2. As $n \rightarrow \infty$ for the Skorokhod topology we have, in law,

$$
y_{n, i}(\cdot) \Rightarrow(2 / \beta)^{1 / 2} b_{x}+x^{2}(i-1), \quad i=1,2 .
$$

Independence of the $i=1,2$ cases now implies (5.2) of Assumption 1.

Lemma 6.3. The bounds (5.4), (5.5) of Assumption 2 hold with $\bar{\eta}(x)=x$.

Proof. There is the estimate

$$
\sqrt{r}(1-4 / r) \leq \mathbb{E} \chi_{r}=\sqrt{2} \frac{\Gamma((r+1) / 2)}{\Gamma(r / 2)} \leq \sqrt{r},
$$

and, again with $\eta_{n, 2, k}=2 \sqrt{n}-2 \beta^{-1 / 2} \mathbb{E} \chi_{\beta(n-k)}$, it follows that

$$
k n^{-1 / 2}-c \leq \eta_{n, 2, k} \leq 2 k n^{-1 / 2}+c,
$$

where $c$ depends on $\beta$ only.

Lastly, for (5.6) of Assumption 2, it suffices to prove a tight random constant bound on

$$
\sup _{k=1 \ldots n / m_{n}} k^{\varepsilon-1} \sup _{\ell=0 \ldots m_{n}}\left|w_{n, i, k m_{n}+\ell}-w_{n, i, k m_{n}}\right|^{2} .
$$

(Notice that the estimate is being done in blocks.) Squaring, replacing the first supremum by a sum, and then taking expectations gives

$$
\sum_{k=1}^{n / m_{n}} \frac{\mathbb{E} \sup _{\ell=0 \ldots m_{n}}\left|w_{n, i, k m_{n}+\ell}-w_{n, i, k m_{n}}\right|^{4}}{k^{2-2 \varepsilon}} \leq \sum_{k=1}^{n / m_{n}} \frac{16 \mathbb{E}\left|w_{n, i,(k+1) m_{n}}-w_{n, i, k m_{n}}\right|^{4}}{k^{2-2 \varepsilon}} .
$$

Here we used the $L^{p}$ maximal inequality for martingales; see Section 2.2 of [18. The expectation is now bounded by a constant independent of $n, k$, and so is the entire sum, as required.

The $\beta$-Laguerre case. Once again, [12] provides a family of tridiagonal " $\beta$-Laguerre ensembles", with explicit eigenvalue densities interpolating between those at $\beta=1,2,4$. Take the $n \times n$ bidiagonal random matrix

$$
W_{n, \kappa}^{\beta}=\frac{1}{\sqrt{\beta}}\left[\begin{array}{ccccc}
\tilde{\chi}_{\beta \kappa} & & & & \\
\chi_{\beta(n-1)} & \tilde{\chi}_{\beta(\kappa-1)} & & & \\
& \ddots & \ddots & & \\
& & \chi_{\beta 2} & \tilde{\chi}_{\beta(\kappa-n+2)} & \\
& & & \chi_{\beta} & \tilde{\chi}_{\beta(\kappa-n+1)}
\end{array}\right] \text {, }
$$


where the entries are all independent $\chi$ variables of the indicated parameter. Here $\kappa \in \mathbb{R}$ and necessarily $\kappa>n-1$. Then, by [12], the eigenvalues of $\left(W_{n, \kappa}^{\beta}\right)^{\dagger}\left(W_{n, \kappa}^{\beta}\right)$ have joint density (1.9).

While the above puts $\kappa>n-1$, the obvious duality reproduces all known real and complex $(\beta=1,2)$ results for any limiting ratio of dimensions $\kappa \rightarrow \infty$ and $n \rightarrow \infty$. This $\beta$ family generalizes the so-called "null" Wishart ensembles, distinguishing the important class of $W \Sigma W^{\dagger}$ type matrices with nonidentity $\Sigma$. For progress on the spectral edge of the latter, consult [2] and [17.

We now proceed with the proof of Theorem 1.4. It suffices to prove the claim along a further subsequence of any given subsequence. This allows us to assume that $\kappa=\kappa(n)$ is an increasing function of $n$, and that $n / \kappa(n) \rightarrow \vartheta \in[0,1]$. Begin with the matrix (6.1), now denoted simply $W_{n}$. The "undressed" ensemble $\beta W_{n}^{\dagger} W_{n}$ has the processes

$$
\begin{array}{r}
\tilde{\chi}_{\beta \kappa}^{2}+\chi_{\beta(n-1)}^{2}, \tilde{\chi}_{\beta(\kappa-1)}^{2}+\chi_{\beta(n-2)}^{2}, \tilde{\chi}_{\beta(\kappa-2)}^{2}+\chi_{\beta(n-3)}^{2}, \ldots \\
\tilde{\chi}_{\beta(\kappa-1)} \chi_{\beta(n-1)}, \tilde{\chi}_{\beta(\kappa-2)} \chi_{\beta(n-2)}, \tilde{\chi}_{\beta(\kappa-3)} \chi_{\beta(n-3)}, \ldots
\end{array}
$$

along the main and off-diagonals, respectively. Up to first order, the top left corner of the matrix $W_{n}^{\dagger} W_{n}$ has $n+\kappa$ on the diagonal, and $\sqrt{n \kappa}$ off-diagonal. That is, the top left corner of

$$
\frac{1}{\sqrt{n \kappa}}\left((\sqrt{n}+\sqrt{\kappa})^{2} I_{n}-W_{n}^{\dagger} W_{n}\right)
$$

is approximately a discrete Laplacian. If time is scaled by $m_{n}^{-1}$, then space will have to be scaled by $m_{n}^{2}$ for this to converge to the continuum Laplacian. Now the desired convergence of drift and noise terms determines, up to constant factors,

$$
m_{n}=\left(\frac{\sqrt{n \kappa}}{\sqrt{n}+\sqrt{\kappa}}\right)^{2 / 3}, \quad H_{n}=\frac{m_{n}^{2}}{\sqrt{n \kappa}}\left((\sqrt{n}+\sqrt{\kappa})^{2} I_{n}-W_{n}^{\dagger} W_{n}\right) .
$$

Now the $y$ 's are defined by formula (5.3) and are just partial sums of shifted and scaled versions of (6.2) and (6.3). That is,

$$
\begin{aligned}
\Delta y_{n, 1, k} & =\frac{m_{n}}{\sqrt{n \kappa}}\left(n+\kappa-\beta^{-1}\left(\chi_{\beta(n-k)}^{2}+\tilde{\chi}_{\beta(\kappa-k+1)}^{2}\right)\right), \\
\triangle y_{n, 2, k} & =\frac{m_{n}}{\sqrt{n \kappa}} 2\left(\sqrt{n \kappa}-\beta^{-1} \chi_{\beta(n-k)} \tilde{\chi}_{\beta(\kappa-k)}\right) .
\end{aligned}
$$

As before, we set $\eta$ to be the expected increments and $w$ to be the centered $y$. The $y_{n, i, .}, i=1,2$ are again independent increment processes, though they are not independent of one another. We set $\gamma=\lim _{n \rightarrow \infty} 2 \sqrt{n / \kappa} /(\sqrt{n / \kappa}+1)^{2} \in[0,1 / 2]$. Then with $x=k / m_{n}$ we have

$$
\begin{aligned}
m_{n} \mathbb{E} \triangle y_{1, n, k} & =\gamma x+o(1), \\
m_{n} \mathbb{E}\left(\triangle y_{1, n, k}\right)^{2} & =\frac{1-\gamma}{2 \beta}+o(1), \quad m_{n} \mathbb{E}\left(\triangle y_{1, n, k}^{4}\right)=o(1),
\end{aligned}
$$

uniformly for $k / m_{n}$ in compacts, so Corollary 6.1 shows that $y_{n, 1}(x)$ converges to the process $(\sqrt{\gamma} / \sqrt{2 \beta}) b_{x}+\gamma x^{2} / 2$, whence it is tight. Similarly we get the convergence process $y_{n, 2}(x) \Rightarrow(\sqrt{1-\gamma} / \sqrt{2 \beta}) b_{x}+(1-\gamma) x^{2} / 2$.

To get the convergence of the sum and (5.4), we instead consider the process defined by

$$
\triangle y_{n, k}=\frac{m_{n}}{\sqrt{n \kappa}}\left((\sqrt{n}+\sqrt{\kappa})^{2}-\beta^{-1}\left(\chi_{\beta(n-k)}+\tilde{\chi}_{\beta(\kappa-k)}\right)^{2}\right),
$$


noting that the process $y_{n, k}-y_{n, 1, k}-y_{n, 2, k}$ is in subscaling and hence converges to the 0 process in law by a fourth moment bound. Now $y_{n, k}$ has independent increments, and the same brand of moment computations already considered along with Corollary 6.1 imply that it converges to $(2 / \sqrt{\beta}) b_{x}+x^{2} / 2$.

Towards tightness, we set $\eta_{n, i, k}=m_{n} \mathbb{E} \Delta y_{i, n, k}$. A bit of work shows that for $\beta$ fixed and all $k \geq 1 \kappa, n>10$ we have

$$
c_{1} \frac{k}{m_{n}} \leq m_{n}\left(\eta_{n, 1, k}+\eta_{n, 2, k}\right) \leq c_{2} \frac{k}{m_{n}} .
$$

We also have the upper bound $m_{n}\left(\eta_{n, 2, k}\right) \leq 2 m_{n}^{2}$. This verifies (5.4) and (5.5) with $\bar{\eta}(x)=x$. The verification of the oscillation bounds (5.6) is identical to the $\beta$-Hermite case. Indeed, all we used there was that $\sqrt{m_{n}} w_{n, i, k}$ are martingales whose increments are independent and have bounded fourth moments.

\section{ACKNOWLEDGMENTS}

The authors would like to thank A. Edelman and B. Sutton for making earlier versions of 15 available to them. Thanks goes as well to M. Krishnapur, H.P. McKean, and B. Valkó for comments and again to L. Dumaz for help with the proof of (4.7).

\section{REFERENCES}

1. J. Baik, P. Deift, K. Johansson, On the distribution of the length of the longest increasing subsequence of random permutations. J. Amer. Math. Soc. 12 (1999), 1119-1178. MR.1682248 (2000e:05006)

2. J. Baik, G. Ben Arous, S. Péché, Phase transition of the largest eigenvalue for non-null complex sample covariance matrices. Ann. Probab. 33 (2005), 1643-1697. MR2165575 (2006g:15046)

3. J. Baik, R. Buckingham, J. DiFranco, Asymptotics of Tracy-Widom distributions and the total integral of a Painlevé II function. Comm. Math. Phys., 280 (2008), 463-497. MR2395479 (2009e:33068)

4. Y. Baryshnikov, GUEs and queues. Probab. Theory Rel. Fields. 119 (2001), 256-274. MR.1818248 (2002a:60165)

5. S. Cambronero, H.P. McKean, The Ground State Eigenvalue of Hill's Equation with White Noise Potential. Comm. Pure Appl. Math. 52 (1999), no 10, 1277-1294. MR1699969 (2001g:60158)

6. S. Cambronero, J. Ramírez, B. Rider, On the shape of the ground state eigenvalue density of a random Hill's equation. Comm. Pure Appl Math. 59 (2006), 935-976. MR2222441 (2007a:34036a)

7. P. Deift, Orthogonal polynomials and random matrices: a Riemann-Hilbert approach. Courant Lecture Notes in Mathematics 3, AMS Providence, RI, 1999. MR.1677884 (2000g:47048)

8. P. Deift, D. Gioev, Random Matrix Theory: invariant ensembles and universality. Courant Lecture Notes in Mathematics 18, AMS Providence, RI, 2009. MR.2514781 (2011f:60008)

9. P. Desrosiers, P. Forrester, Hermite and Laguerre $\beta$-ensembles: asymptotic corrections to the eigenvalue density. Nuc. Phys. B. 743 (2006), 307-332. MR.2227950 (2007f:82043)

10. M. Dieng, Distribution functions for edge eigenvalues in Orthogonal and Symplectic Ensembles: Painlevé representations. Int. Math. Res. Notices. 2005, 2263-2287. MR 2181265 (2006h:60014)

11. I. Dumitriu, Personal communication, 2006.

12. I. Dumitriu, A. Edelman, Matrix models for beta ensembles. J. Math. Phys. 43 (2002), 58305847. MR $1936554(2004 \mathrm{~g}: 82044)$

13. L. Dumaz, The Tracy-Widom right tail. Master's thesis, Ecole Normale Supérieure, 2009.

14. L. Dumav, B. Virág The right tail exponent of the Tracy-Widom-beta distribution. Preprint, arXiv:1102.4818 (2011). 
15. A. Edelman, B. Sutton, From random matrices to stochastic operators. J. Stat. Phys. 127 (2007), 1121-1165. MR2331033 (2009b:82037)

16. N. El Karoui, On the largest eigenvalue of Wishart matrices with identity covariance when $n, p$, and $p / n \rightarrow \infty$. Preprint, arXiv:math.ST/0309355 (2003).

17. N. El Karoui, Tracy-Widom limit for the largest eigenvalue of a large class of complex Wishart matrices. Ann. Probab. 35 (2007), 663-714. MR2308592 (2007m:60057)

18. S. Ethier, T. Kurtz, Markov processes, characterization and convergence, Wiley, 1986. MR838085 (88a:60130)

19. P. Ferrari, H. Spohn, Scaling limit for the space-time covariance of the stationary totally asymmetric simple exclusion process. Comm. Math. Phys. 265 (2006), 1-44. MR 2217295 (2007g:82038a)

20. P. Forrester, Log-Gases and Random Matrices. London Math. Soc. Monographs, Princeton University Press, 2010. MR.2641363 (2011d:82001)

21. B.I. Halperin, Green's functions for a particle in a one-dimensional random potential. Phys. Rev. (2) 139 (1965), A104-A117. MR0187859 (32:5304)

22. K. Johansson, Shape fluctuations and random matrices. Comm. Math. Phys. 209 (2000), 437-476. MR 1737991 (2001h:60177)

23. I.M. Johnstone, On the distribution of the largest eigenvalue in principal components analysis. Ann. Statist. 29 (2001), 295-327. MR1863961 (2002i:62115)

24. S. Killip, M. Stoiciu, Eigenvalue Statistics for CMV Matrices: From Poisson to Clock via Random Matrix Ensembles. Duke Math. Journal 146 (2009), 361-399. MR2484278 (2009k:81087)

25. H.P. McKean, A limit law for the ground state of Hill's equation. J. Stat. Phys. 74 (1994), 1227-1232. MR1268791 (95d:82036)

26. M. Prähofer, H. Spohn, Scale invariance of the PNG droplet and the Airy process. J. Stat. Phys. 108 (2002), 1071-1106. MR.1933446(2003i:82050)

27. J. Ramírez, B. Rider, Diffusion at the random matrix hard edge. Comm. Math. Phys. 288 (2009), 887-906 MR2504858 (2010g:47083)

28. B.D. Sutton, The stochastic operator approach to random matrix theory. Ph.D. thesis, MIT, Department of Mathematics, 2005. MR2717319

29. C. Tracy, H. Widom, Level spacing distributions and the Airy kernel. Comm. Math. Phys. 159 (1994), 151-174. MR.1257246 (95e:82003)

30. C. Tracy, H. Widom On orthogonal and symplectic matrix ensembles. Comm. Math. Phys. 177 (1996), 727-754. MR1385083 (97a:82055)

31. H. F. Trotter,(1984) Eigenvalue distributions of large Hermitian matrices; Wigner's semicircle law and a theorem of Kac, Murdock, and Szegö. Adv. in Math. 54 (1984), 67-82. MR761763 (86c:60055)

32. B. Valko, B. Virág, Continuum limits of random matrices and the Brownian carousel. Inventiones 177 (2009), 463-508. MR2534097(2011d:60023)

33. B. Valko, B. Virág, Large gaps between random eigenvalues. Ann. Probab. 38 (2010), 12631279. MR2674999

Department of Mathematics, Universidad de Costa Rica, San Jose 2060, Costa Rica E-mail address: alexander.ramirez_g@ucr.ac.cr

Department of Mathematics, University of Colorado at Boulder, UCB 395, Boulder, COLORADO 80309

E-mail address: brian.rider@colorado.edu

Department of Mathematics and Statistics, University of Toronto, Ontario, M5S 2E4, CANADA

E-mail address: balint@math.toronto.edu 